\title{
Inhibiting the MNK1/2-elF4E axis impairs melanoma phenotype switching and potentiates antitumor immune responses
}

Fan Huang, ${ }^{1,2}$ Christophe Gonçalves, ${ }^{1}$ Margarita Bartish, ${ }^{1,2}$ Joelle Rémy-Sarrazin, ${ }^{2}$ Mark E. Issa, ${ }^{3}$ Brendan Cordeiro, ${ }^{4}$ Qianyu Guo,,${ }^{1,2}$ Audrey Emond, ${ }^{1}$ Mikhael Attias, ${ }^{4,5}$ William Yang, ${ }^{1,2}$ Dany Plourde, ${ }^{1}$ Jie Su, ${ }^{1}$ Marina Godoy Gimeno, ${ }^{6}$ Yao Zhan, ${ }^{1,2}$ Alba Galán, ${ }^{1}$ Tomasz Rzymski, ${ }^{7}$ Milena Mazan, ${ }^{7}$ Magdalena Masiejczyk, ${ }^{7}$ Jacek Faber, ${ }^{7}$ Elie Khoury, ${ }^{1,2}$ Alexandre Benoit, ${ }^{1,2}$ Natascha Gagnon, ${ }^{1}$ David Dankort, ${ }^{8,9}$ Fabrice Journe, ${ }^{10}$ Chanem E. Chanem, ${ }^{10}$ Connie M. Krawczyk, ${ }^{4}$ H. Uri Saragovi, ${ }^{1,11}$ Ciriaco A. Piccirillo, ${ }^{4,5}$ Nahum Sonenberg, ${ }^{9,12}$ Ivan Topisirovic, ${ }^{1,2,13}$ Christopher E. Rudd, ${ }^{3,13}$ Wilson H. Miller Jr., ${ }^{1,2,13}$ and Sonia V. del Rincón ${ }^{1,2,13}$

'Lady Davis Institute, Jewish General Hospital, Montréal, Quebec, Canada. ²Division of Experimental Medicine, McGill University, Montréal, Quebec, Canada. ${ }^{3}$ Maisonneuve-Rosemont Hospital Research Centre, Montréal, Quebec, Canada. ${ }^{4}$ Department of Microbiology and Immunology and ${ }^{5}$ Research Institute of the McGill University Health Centre, McGill University, Montréal, Quebec, Canada. ${ }^{6}$ University Veterinary Teaching Hospital Camden, Faculty of Science, University of Sydney, Sydney, New South Wales, Australia. ${ }^{7}$ Ryvu Therapeutics, Kraków, Poland. ${ }^{8}$ Department of Biology and ${ }^{9}$ Coodman Cancer Research Center, McGill University, Montréal, Quebec, Canada. ${ }^{10}$ Laboratory of Oncology and Experimental Surgery, Institut Jules Bordet, Université Libre de Bruxelles, Brussels, Belgium. ${ }^{11 D e p a r t m e n t ~ o f ~}$ Pharmacology and Therapeutics, ${ }^{12}$ Department of Biochemistry, and ${ }^{13}$ McCill Centre for Translational Research in Cancer, McGill University, Montréal, Quebec, Canada.

\begin{abstract}
Melanomas commonly undergo a phenotype switch, from a proliferative to an invasive state. Such tumor cell plasticity contributes to immunotherapy resistance; however, the mechanisms are not completely understood and thus are therapeutically unexploited. Using melanoma mouse models, we demonstrated that blocking the MNK1/2-elF4E axis inhibited melanoma phenotype switching and sensitized melanoma to anti-PD-1 immunotherapy. We showed that phosphoelF4E-deficient murine melanomas expressed high levels of melanocytic antigens, with similar results verified in patient melanomas. Mechanistically, we identified phospho-elF4E-mediated translational control of NGFR, a critical effector of phenotype switching. Genetic ablation of phospho-elF4E reprogrammed the immunosuppressive microenvironment, exemplified by lowered production of inflammatory factors, decreased PD-L1 expression on dendritic cells and myeloidderived suppressor cells, and increased CD8 ${ }^{+}$T cell infiltrates. Finally, dual blockade of the MNK1/2-elF4E axis and the PD-1/ PD-L1 immune checkpoint demonstrated efficacy in multiple melanoma models regardless of their genomic classification. An increase in the presence of intratumoral stem-like TCF1+PD-1+CD8 + T cells, a characteristic essential for durable antitumor immunity, was detected in mice given a MNK1/2 inhibitor and anti-PD-1 therapy. Using MNK1/2 inhibitors to repress phospho-elF4E thus offers a strategy to inhibit melanoma plasticity and improve response to anti-PD-1 immunotherapy.
\end{abstract}

\section{Introduction}

Malignant melanoma is the deadliest form of skin cancer. In melanoma, the major signaling pathways, RAS/RAF/MAPK and $\mathrm{PI} 3 \mathrm{~K} / \mathrm{AKT}$, are constitutively activated through numerous avenues, including genetic alterations in BRAF and PTEN, respectively. Both pathways ultimately converge upon eukaryotic translation initiation factor $4 \mathrm{E}$ (eIF4E) to induce its phosphorylation. $B R A F^{V 600 E}$ is the most common activating mutation in cutaneous melanoma and is upstream of MAP kinase-interacting serine/ threonine-protein kinase 1 (MNK1) and MNK2, which directly phosphorylate eIF4E (1-3). Loss-of-function mutations in PTEN, occurring in up to $30 \%$ of patients with BRAF-mutant melanoma, on the other hand, will indirectly result in the phosphorylation of

Conflict of interest: The authors have declared that no conflict of interest exists. Copyright: () 2021, American Society for Clinical Investigation.

Submitted: May 28, 2020; Accepted: March 3, 2021; Published: April 15, 2021

Reference information: J Clin Invest. 2021;131(8):e140752.

https://doi.org/10.1172/JCl140752.
eIF4E via activation of the mTOR/4E-BP1 axis (1). Many groups, including our own, have shown that dysregulated mRNA translation by aberrant activation of the MNK1/2-eIF4E axis plays a critical role in tumor progression to invasive and metastatic disease (4-10). Moreover, phospho-eIF4E expression is increased during melanoma progression $(4,5)$. The phosphorylation of eIF4E on serine 209 (S209), catalyzed exclusively by MNK1 and MNK2 $(2,3)$, increases the oncogenic potential of eIF4E (6-8). Mechanistically, phospho-eIF4E selectively increases the translation of a subset of mRNAs encoding proteins involved in cell survival, proliferation, and metastasis (8-11).

Immunotherapies blocking immune checkpoints, such as CTLA-4 and the PD-1/PD-L1 axis, show therapeutic efficacy in patients with metastatic melanoma (12-15). Antibodies against CTLA-4, PD-1, or PD-L1 are currently approved for clinical use, or are in clinical trials, in many types of cancers (16-18). Despite the approval of immunotherapy for the management of a growing number of cancer types, checkpoint blockade as monotherapy has achieved limited clinical success in most malignancies. Many 
A

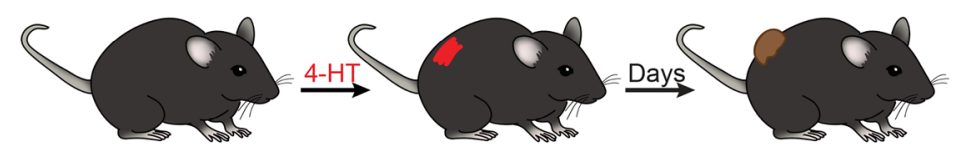

Alleles

Tyr::CreER CRE ER

BRaf ${ }^{C A+} \quad$ BRAF ${ }^{W T}$

Pten 10x10x PTEN WT

Eif4e WTNT elF4E WT

Eif4e ${ }^{\text {KIKI }}$ elF4E $2209 \mathrm{~A}$

Proteins expressed

elF4E WT

elF4E S209A
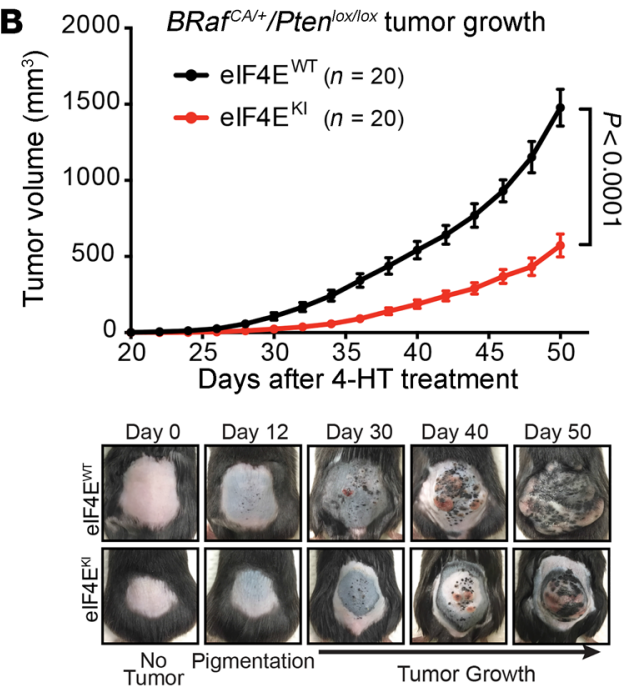

C

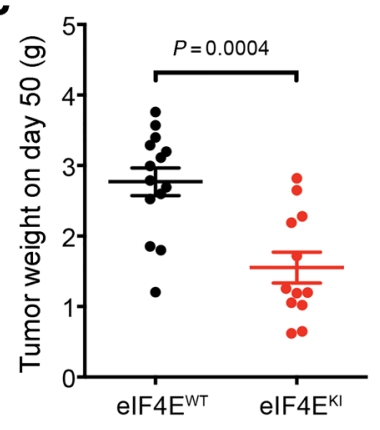

$\mathbf{F}$

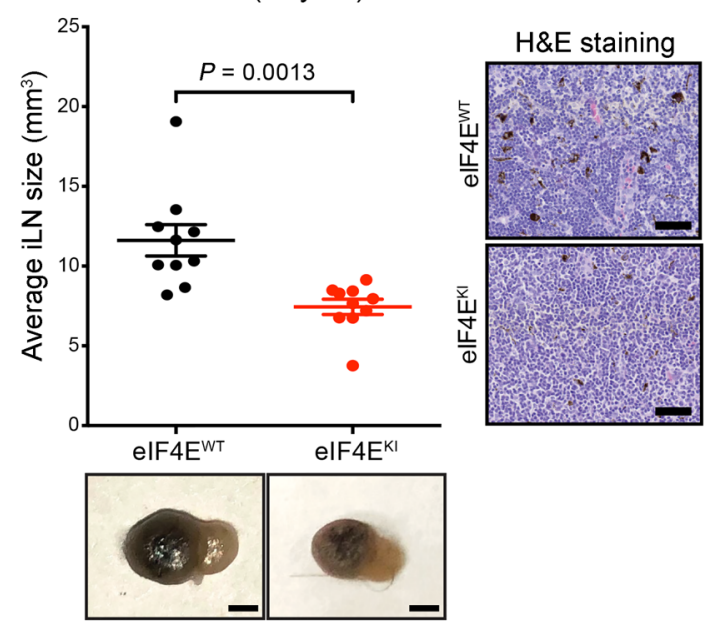

D
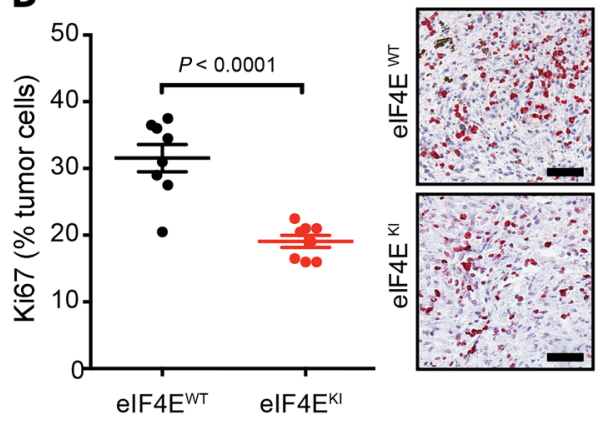

G

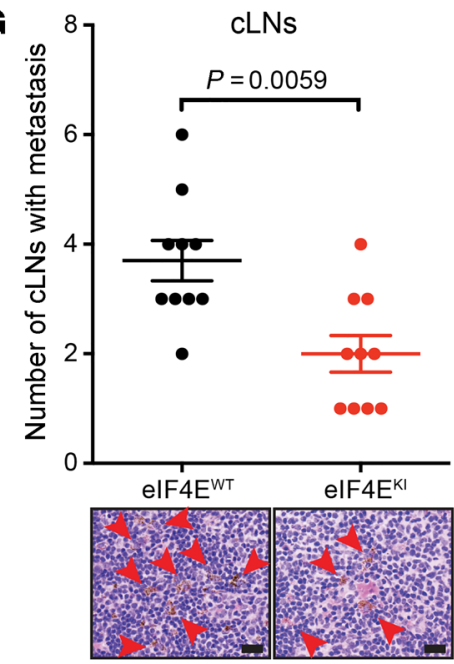

cLNs
E

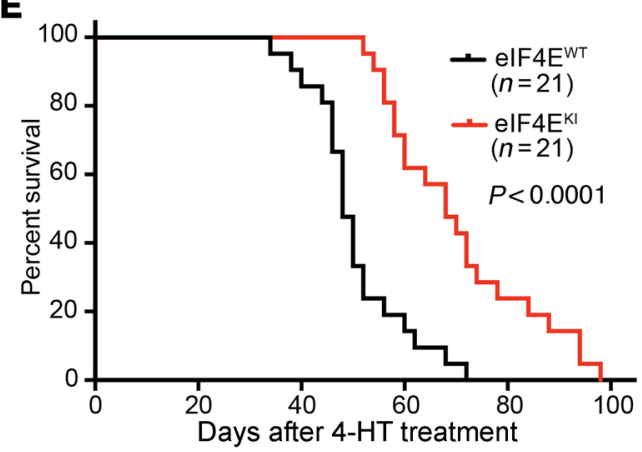


patients fail to respond, and others who initially had responded eventually experience tumor relapse $(18,19)$. Therapeutic efficacy of combining inhibitors against CTLA- 4 and PD- 1 can also be hampered by the appearance of life-threatening immune-related adverse events (20). Key factors that favor a response to immune checkpoint blockade include the presence of immune cell infiltration and the availability of tumor-associated target antigens (2125). Melanoma plasticity, exhibited as phenotype switching, has been proposed to contribute to primary or secondary resistance to immunotherapy and is a process akin to the epithelial-mesenchymal transition (26-31). During phenotype switching, melanomas undergo dedifferentiation with (a) a loss of melanocytic antigens, (b) increased proinflammatory cytokine/chemokine secretion, and (c) tumor infiltration of myeloid-derived suppressor cells (2635). However, the mechanisms underlying the regulation of the phenotype switch are not completely understood, and thus, few therapeutic approaches have been exploited to prevent or block this process (26).

Here, we investigated the role of the MNK1/2-eIF4E axis in melanoma progression and antitumor immunity. We hypothesized that phospho-eIF4E promotes melanoma phenotype switching and metastasis and functions also in non-melanoma cells to support an immunosuppressive microenvironment that restricts robust antitumor responses to immunotherapy. MNK1/2 inhibitors, blocking the phosphorylation of eIF4E, therefore serve a dual purpose as (a) therapies that inhibit tumor plasticity and (b) immunomodulatory agents that can be used in combination with immunotherapies in metastatic melanoma.

\section{Results}

Phospho-eIF4E promotes melanoma outgrowth and metastasis. To determine whether phospho-eIF4E contributes to melanoma development and metastasis in vivo, we crossed eIF4E $\mathrm{E}^{\mathrm{S} 209 \mathrm{~A} / \mathrm{S} 209 \mathrm{~A}}$ mice, in which eIF4E cannot be phosphorylated (7), with the well-described Tyr::CreER/BRafCA/+ $/$ Pten $^{\text {lox } / \text { lox }}$ conditional melanoma model $(36,37)$. This melanoma model allows 4-hydroxytamoxifen-inducible (4-HT-inducible) melanocyte-targeted $B R A F^{V 600 E}$ expression and simultaneous PTEN inactivation (Figure 1A). As expected, following topical administration of 4-HT on the lower back of mice, hyperpigmented lesions were observed within 12-15 days, and melanomas developed within 2-3 weeks (Figure 1B). Compared with the BRafCA/+ $/$ Pten $^{\text {lox } / \text { lox }}$ (referred to hereafter as eIF4E $\mathrm{E}^{\mathrm{WT}}$ ) mice, the melanoma outgrowth in $B R a f^{\mathrm{CA} /+} /$ Pten $^{\text {lox/lox }} /$ Eif $e^{\text {S209A/S209A }}$ (henceforth termed eIF4E $\mathrm{E}^{\mathrm{KI}}$ ) mice, devoid of phosphorylated eIF4E, was robustly decreased (Figure 1, B and C). While we did not observe any significant difference in tumor initiation between the eIF $4 \mathrm{E}^{\mathrm{WT}}$ and $\mathrm{eIF} 4 \mathrm{E}^{\mathrm{KI}}$ mice (Supplemental Figure 1A; supplemental material available online with this article; https://doi.org/10.1172/JCI140752DS1), Ki67 staining revealed a less proliferative state of the eIF4 $\mathrm{E}^{\mathrm{KI}}$ melanomas (Figure 1D), indicating that phospho-eIF4E promotes melanoma proliferation in vivo. The protection against primary melanoma outgrowth is dependent on the dosage of phospho-eIF4E loss, as mice expressing 1 copy of eIF $4 \mathrm{E}$ and 1 copy of eIF $4 \mathrm{E}^{\mathrm{S} 209 \mathrm{~A}}\left(\mathrm{eIF} 4 \mathrm{E}^{\mathrm{HET}}\right)$ exhibited a delay in melanoma outgrowth compared with eIF4E $\mathrm{E}^{\mathrm{WT}}$ mice (Supplemental Figure 1B). Importantly, eIF4E $\mathrm{E}^{\mathrm{KI}}$ mice exhibited a significant increase in survival compared with their eIF4 $4 \mathrm{E}^{\mathrm{WT}}$ coun- terparts (Figure 1E). In this mouse model, melanomas metastasized to the draining inguinal lymph nodes (iLNs), which enlarged throughout tumor progression (Supplemental Figure 1C). However, the observed iLN enlargement was hampered in the eIF $4 \mathrm{E}^{\mathrm{KI}}$ mice (Figure 1F and Supplemental Figure 1C). Moreover, 3 weeks after tumor induction, when there was no significant difference in iLN size between the 2 genotypes (Supplemental Figure 1C), we detected iLN metastasis in the eIF4E $\mathrm{E}^{\mathrm{WT}}$ mice, but not in eIF4E $4 \mathrm{E}^{\mathrm{KI}}$ mice (Supplemental Figure 1, D and E). Finally, metastasis to the distant cervical lymph nodes (cLNs) was significantly decreased in the eIF $4 \mathrm{E}^{\mathrm{KI}}$ mice compared with the eIF $4 \mathrm{E}^{\mathrm{WT}}$ mice, assessed when primary melanomas were size-matched between $500 \mathrm{~mm}^{3}$ and $800 \mathrm{~mm}^{3}$ (Figure $1 \mathrm{G}$ ). These results suggest that inhibiting the phosphorylation of eIF4E profoundly hinders melanoma outgrowth and metastasis.

Inhibition of phospho-eIF4E blocks melanoma dedifferentiation and loss of melanocytic antigens. We next characterized the histology of melanomas derived from eIF $4 \mathrm{E}^{\mathrm{WT}}$ and $\mathrm{eIF} 4 \mathrm{E}^{\mathrm{KI}}$ transgenic mice. Upon inspection of the H\&E-stained primary melanomas, while no marked morphological differences were observed, we noted a significant increase in melanin pigmentation in the eIF4 $\mathrm{E}^{\mathrm{KI}}$ melanomas as compared with eIF4E $\mathrm{E}^{\mathrm{WT}}$ melanomas (Figure 2A and Supplemental Figure 2A). In addition, immunohistochemical staining for the melanoma marker S100 showed that the frequency of pigmented S100-positive melanoma cells was significantly higher in the eIF$4 \mathrm{E}^{\mathrm{KI}}$ cohort compared with the eIF4 $\mathrm{E}^{\mathrm{WT}}$ cohort (Figure 2B). Melanin pigmentation is tightly controlled by the microphthalmia-associated transcription factor (MITF), which is critical for the survival of pigmented cells and drives melanocyte differentiation (38). Dedifferentiated melanomas, characterized by a loss, or low expression, of MITF, have been associated with increased invasion and metastasis, therapeutic resistance, and reduced overall patient survival $(27,28)$. eIF $4 \mathrm{E}^{\mathrm{KI}}$ melanomas expressed more MITF compared with eIF $4 \mathrm{E}^{\mathrm{WT}}$ melanomas (Figure 2C). Moreover, the expression of 2 downstream melanocytic differentiation antigens, Melan-A (also known as MART-1) and GP100 (also known as Pmel17) $(39,40)$, was significantly increased in the eIF $4 \mathrm{E}^{\mathrm{KI}}$ melanomas (Figure 2, D and E). Consistent with previous studies (37, 41, 42), Melan-A expression was virtually undetectable in most of the eIF4 $\mathrm{E}^{\mathrm{WT}}$ melanomas (Figure 2D and Supplemental Figure 2B). Melanomas harboring a $50 \%$ reduction in phospho-eIF4E (eIF4 $\mathrm{E}^{\mathrm{HET}}$ ) also showed increased Melan-A expression relative to eIF4 $\mathrm{E}^{\mathrm{WT}}$ melanomas (Supplemental Figure 2B). In support of the clinical relevance of our findings, the expression of phospho-eIF4E was negatively correlated with the expression of Melan-A in patient-derived melanomas (Figure 2F). Human melanomas with a high overall expression of phospho-eIF4E had significantly lower expression or, in many patient samples, no Melan-A expression. Conversely, melanomas with low overall phospho-eIF4E expression showed intense Melan-A staining (Figure $2 \mathrm{~F})$. Intriguingly, in patient samples with regional phospho-eIF4E expression, the staining for phospho-eIF4E and that for Melan-A were mutually exclusive (Supplemental Figure 2C). Together, these data suggest that repressing the phosphorylation of eIF4E in melanoma results in a differentiated phenotype characterized by the retention of pigmentation and melanocytic antigens.

We next reasoned that blocking the activity of MNK1 and MNK2, the kinases that phosphorylate eIF4E, would reverse 
A

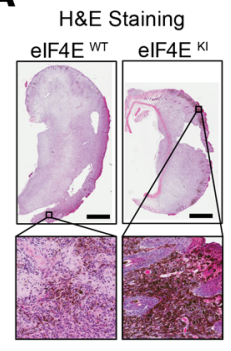

B

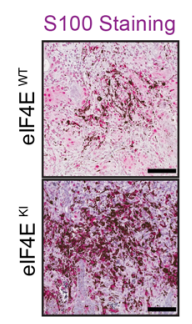

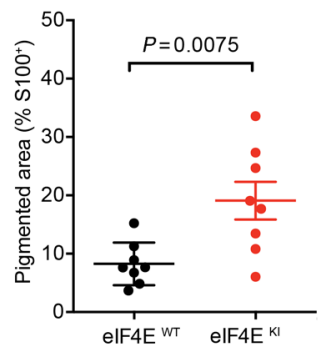

D
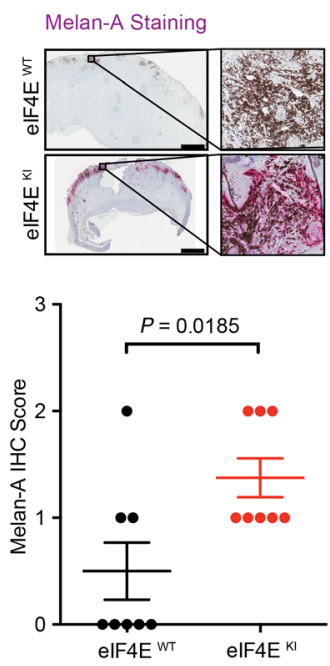

E
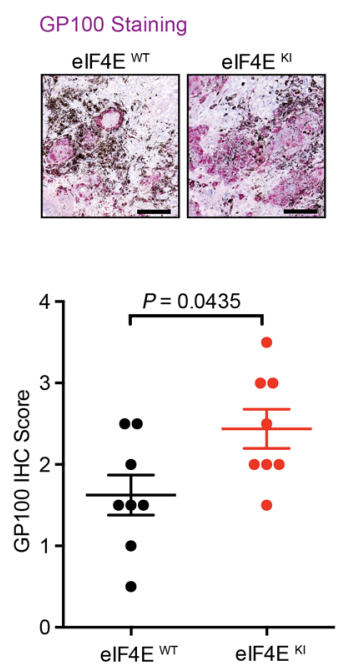

C elF4E WT elF4E ${ }^{\mathrm{KI}}$

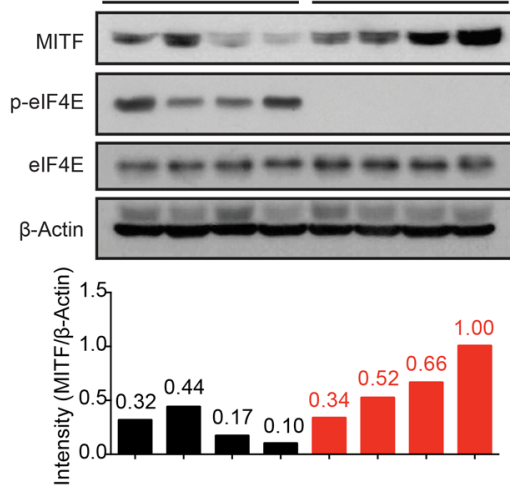

$\mathbf{F}$

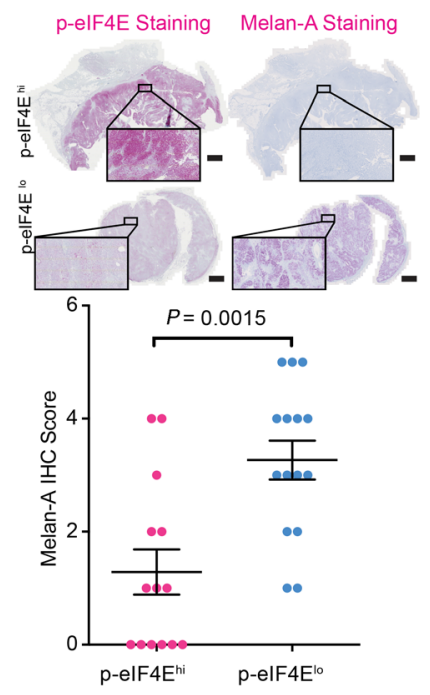

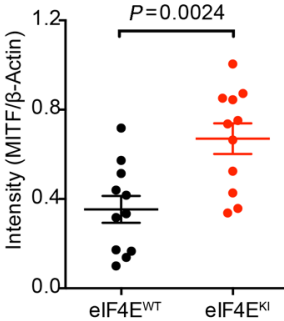

G

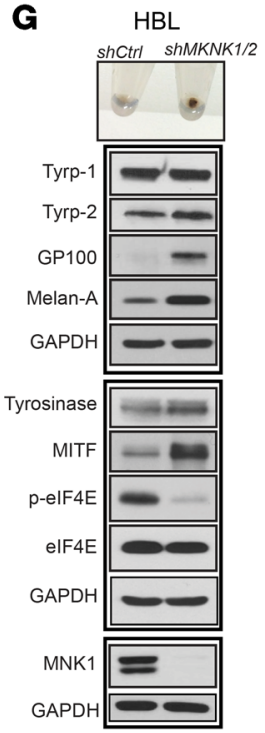

Figure 2. Phospho-elF4E-deficient murine and human melanomas are more differentiated. (A) Representative elF4E ${ }^{\mathrm{WT}}(n=16)$ and elF4E primary tumor histology with H\&E staining. (B) Representative IHC images showing the expression of S100 (left) and the percentage of pigmented area

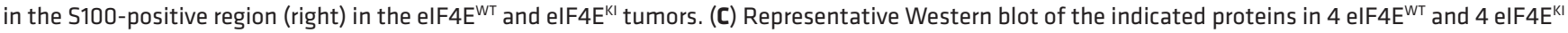
melanomas (top left; see all blots in Supplemental Figure 12) with quantification of MITF level normalized to $\beta$-actin (bottom left and right). (D and $\mathbf{E}$ ) Representative IHC images with scores showing the expression of Melan-A (D) and GP100 (E) in elF4EWT and elF4E melanomas. (F) Top: IHC images showing the expression of phospho-elF4E and Melan-A in 2 representative tumors from a total of 29 patients with melanoma. One patient sample with high phospho-elF4E expression (score $>2.5 / 5$ ) and one patient sample with low phospho-elF4E expression (score $=0.5-1 / 5)$ are shown. Bottom: IHC scoring of Melan-A in phospho-elF4E $\mathrm{E}^{\mathrm{hi}}$ and phospho-elF4E ${ }^{\mathrm{lo}}$ patient-derived melanomas. (C) Western blot analysis of the indicated proteins in human $\mathrm{HBL}$ melanoma cells stably expressing shCtrl and shMKNK1/2 (representative of $n=3$ ). (A-E) Melanomas were resected on day 50. (A, D, and F) Scale bars: $2 \mathrm{~mm}$. (B and E) Scale bars: $100 \mu \mathrm{m}$. (B-F) Number of biological replicates is indicated in each graph. (B and C) Two-sided unpaired $t$ test. (D-F) Mann-Whitney test. All values are represented as mean \pm SEM.

tumor cell plasticity in human melanoma cells. To test this, we used the invasive patient-derived melanoma cell line HBL, wherein we previously knocked down MKNK1 and MKNK2 using shRNAs (9). MKNK1/2 knockdown in HBL cells showed a reduced phospho-eIF4E level concomitant with increased pigmentation and MITF expression (Figure $2 \mathrm{G}$ ). Silencing of $M K N K 1 / 2$ in HBL cells also resulted in an induction of the melanogenic proteins tyrosinase, Tyrp-2, Melan-A, and GP100, as compared with their shRNA control counterparts (Figure 2G). Together, these data suggest that inhibition of the MNK1/2-eIF4E axis reverses melanoma plasticity toward a differentiated phenotype.

Phospho-eIF4E-deficient melanomas are less invasive through inhibition of NGFR. Melanomas that have undergone dedifferentiation, or phenotype switching, are characterized by a more inva- sive phenotype (27, 43-46). Having shown that the mice harboring more differentiated eIF $4 \mathrm{E}^{\mathrm{KI}}$ melanomas also had decreased lymph node metastasis (Figure 1G and Supplemental Figure 1E), we next wanted to recapitulate this phenotype in vitro. We derived murine melanoma cell lines, MDMel-WT and MDMel-KI, from

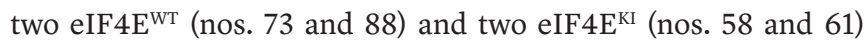
tumor-bearing animals, respectively. All cell lines were confirmed as PTEN negative (Pten ${ }^{l o x} /(0 x)$ and Tyr::CreER positive, similar to D4M.3a murine melanoma cells previously derived from the eIF$4 \mathrm{E}^{\mathrm{WT}}$ mouse model (41) (Figure 3A and Supplemental Figure 3, A and F). Characterization of these cell lines revealed distinct phenotypes. The MDMel-KI cell lines, which are phospho-eIF4E deficient, failed to invade as efficiently as the MDMel-WT cells (Figure $3 \mathrm{~B}$ and Supplemental Figure 3B), despite being more proliferative 
A

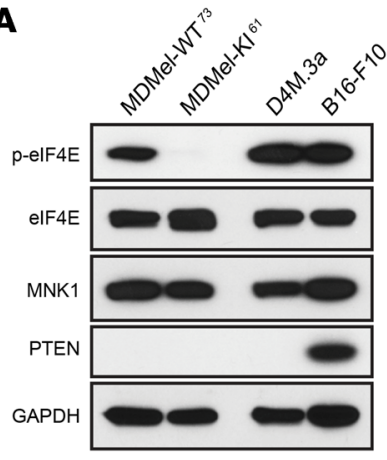

B

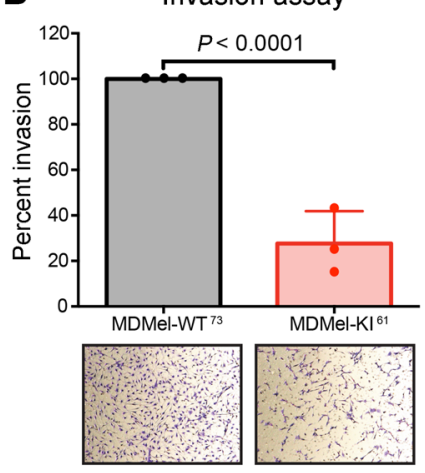

C

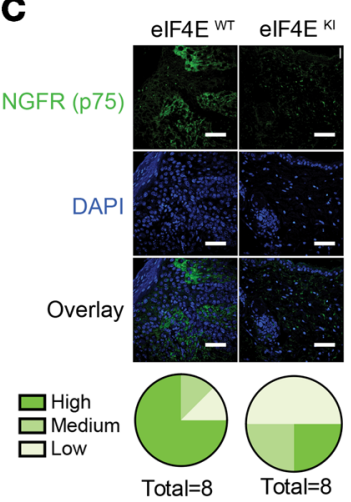

D

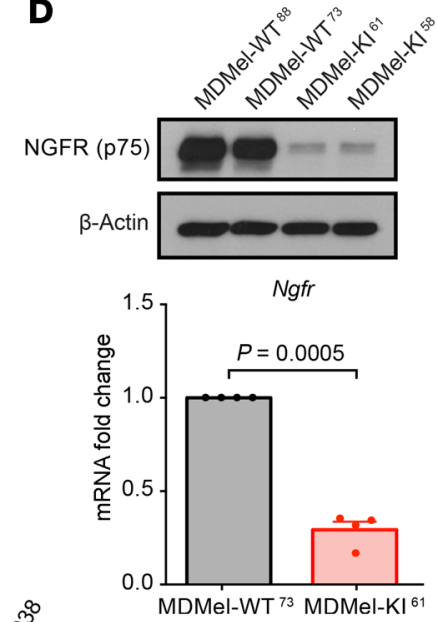

E

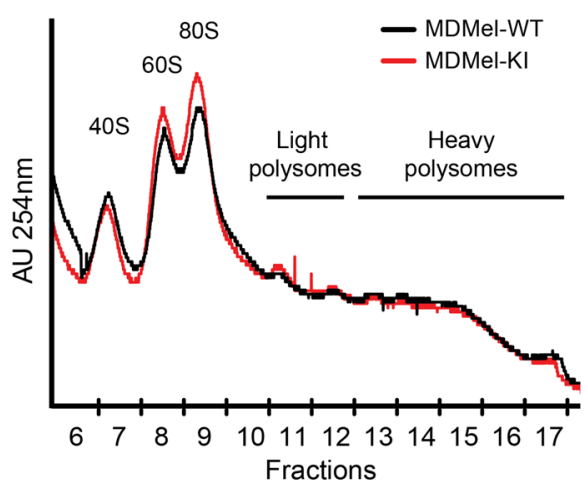

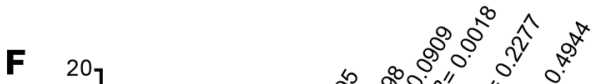

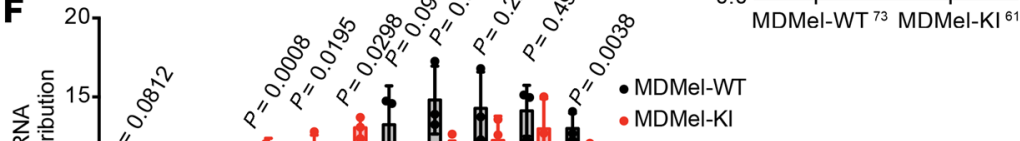

G

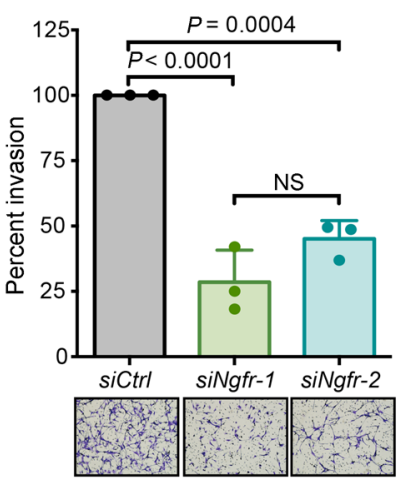

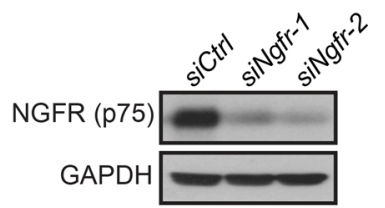

$\mathbf{H}$
Invasion assay
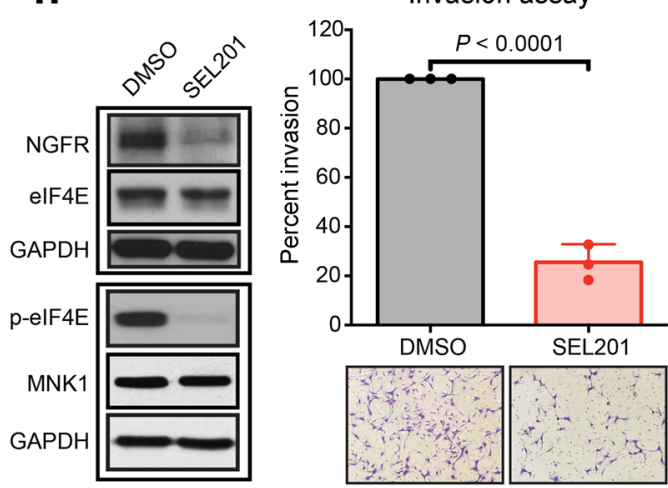

Figure 3. Phospho-elF4E-deficient melanoma cells resist phenotype switching via inhibiting NGFR mRNA translation. (A) Western blot analysis of the indicated proteins in murine melanoma cell lines MDMel-WT ${ }^{73}$, MDMel-KI ${ }^{61}$, D4M.3a, and B16-F10 (representative of $n=3$ ). (B) Percent invasion of MDMel-KI' ${ }^{61}$ cells relative to $\mathrm{MDMel}-\mathrm{WT}^{73}$ cells (top) and representative images (bottom; original magnification, $\times 10 ; n=3$ ). Two-way ANOVA with Tukey's test (comparing all groups shown in Supplemental Figure 3B). (C) Representative immunofluorescence images showing the expression of NGFR in elF4E ${ }^{W T}$ and elF4E ${ }^{\mathrm{Kl}}$ tumors (day 50; top; $n=8$ per group; scale bars: $30 \mu \mathrm{m}$ ) and the percentage of samples with high, medium, and low expression of NGFR from each group (bottom). (D) Top: Western blot analysis of NGFR expression in the murine melanoma cell

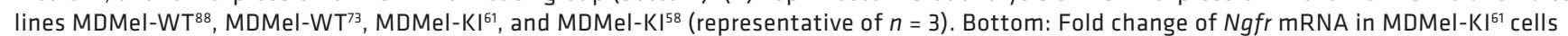
relative to MDMel-WT73 cells, normalized to m36B4 $(R p / p O)$ as a reference gene $(n=4)$. Two-way ANOVA with Tukey's test (comparing all groups shown in Supplemental Figure 3F). (E) Polysome profiles of MDMel-WT and MDMel-KI cells (representative of $n=3)$. (F) Percentage of transcripts in each polysomal fraction quantified by quantitative real-time PCR (top) and representative image showing rRNAs and PCR-amplified cDNA fragments of the indicated targets (bottom; $n=3$ ). Multiple unpaired 2-tailed $t$ test. (G) Left: Percent invasion of MDMel-WT ${ }^{73}$ cells with $\mathrm{Ngfr}$ knockdown relative to the control group (top), and representative images (bottom; original magnification, $\times 10$; see also Supplemental Figure 3G). One-way ANOVA. Right: Western blot confirming knockdown of NGFR $(n=3)$. (H) Left: Western blot analysis of the indicated proteins in DMSOor SEL201-treated MDMel-WT cells. Right: Percent invasion of SEL201-treated cells relative to control (top), and representative images (bottom; original magnification, $\times 10$; representative of $n=3$ ). Two-sided unpaired $t$ test. All values are represented as mean $\pm S D$. 
A

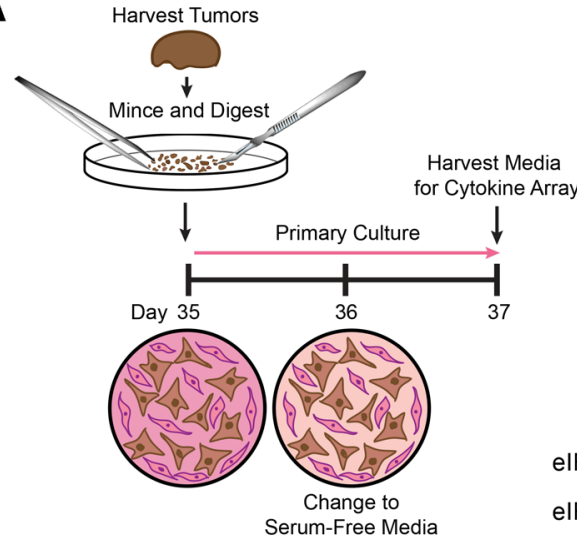

B

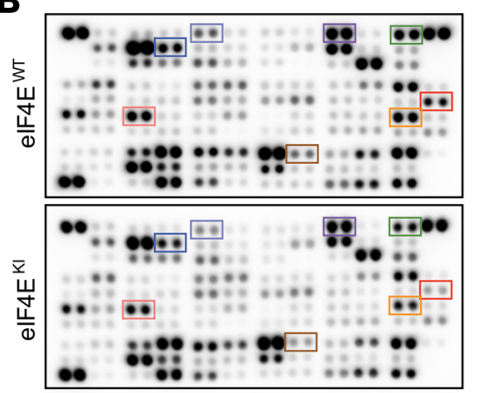

ANGPT2 CCL2 CCL5 CCL12 IGFBP-2 IGFBP-6 IL-6 MMP-9
C

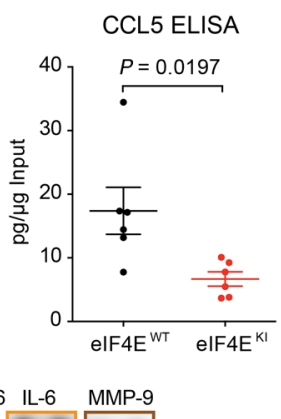

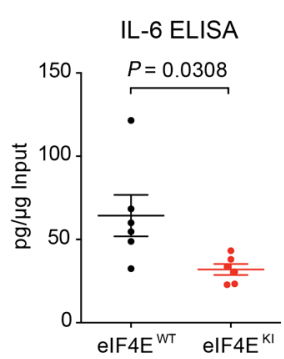

elF4E

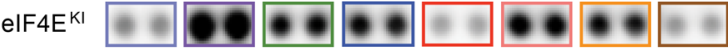
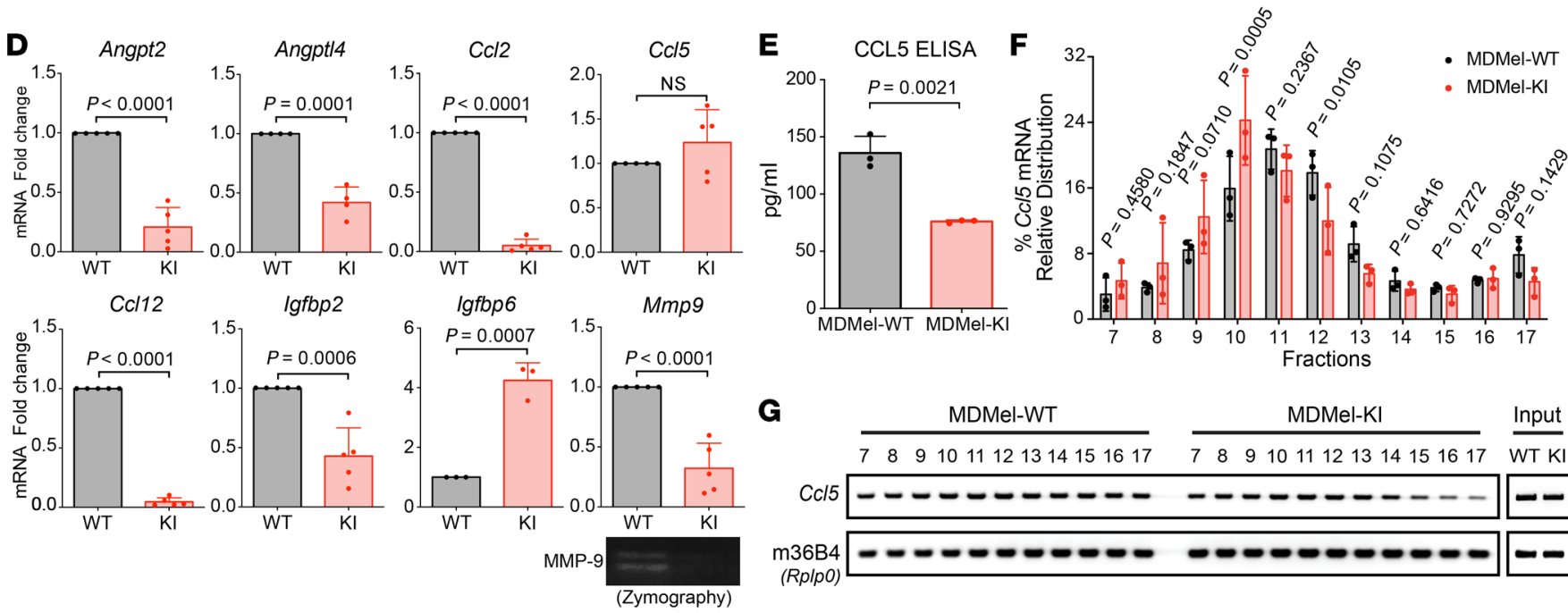

G
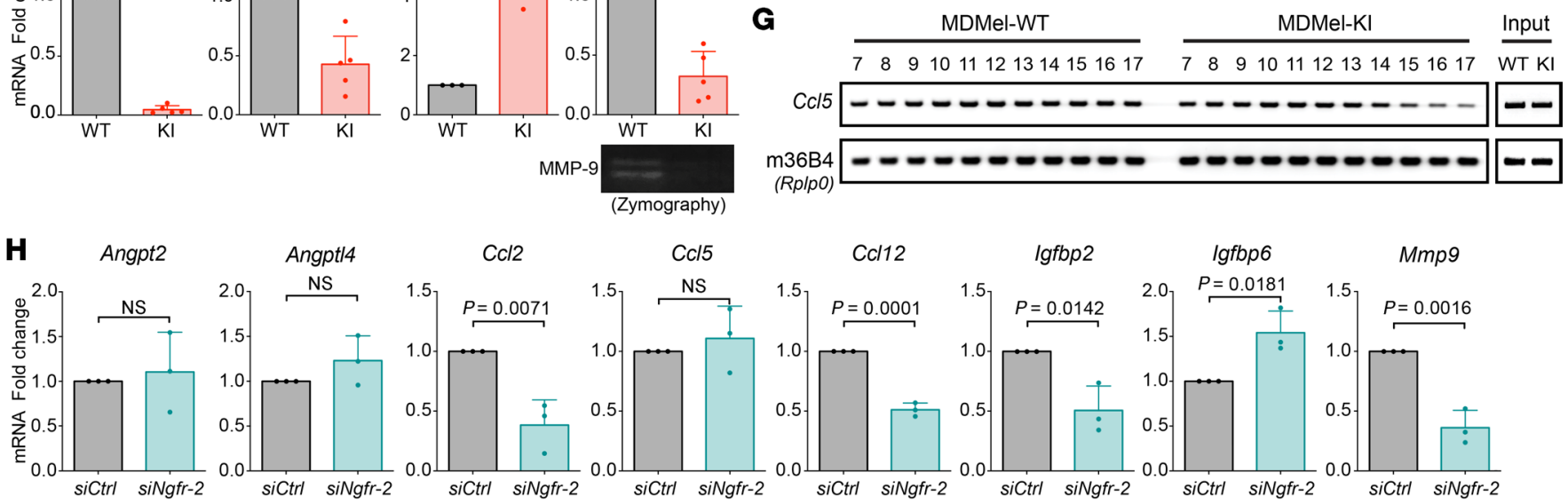

I

Phospho-elF4E-Regulated Cytokines/Chemokines

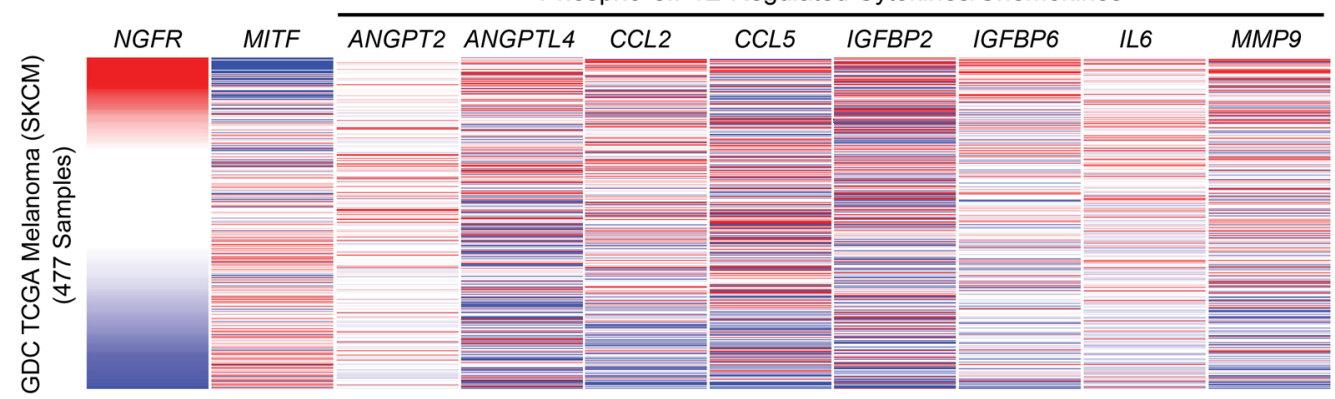

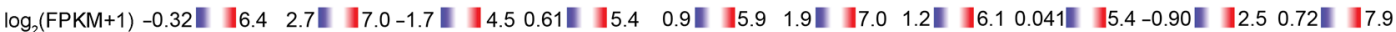

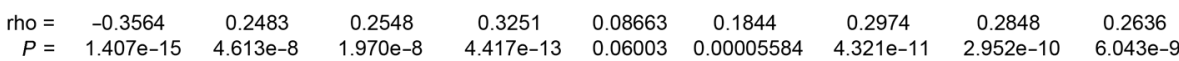


Figure 4. Phospho-elF4E-deficient melanomas have an altered secretome. (A) Schematic of the experimental design for the membrane-based cytokine arrays. (B) Representative images of the cytokine arrays showing the expression of secreted factors present in the conditioned medium derived from elF4E $4 \mathrm{ET}^{\mathrm{TT}}$ and elF4E $\mathrm{E}^{\mathrm{KI}}$ primary melanoma cultures $(n=2$ mice per genotype). (C) Concentration of CCL5 and IL-6 in the conditioned medium derived from the elF4E ${ }^{\mathrm{WT}}$ and elF4E $\mathrm{E}^{\mathrm{Kl}}$ primary melanoma cultures $(n=$ 6 mice per genotype). (D) Fold change of the indicated mRNAs in MDMel$\mathrm{KI}$ cells relative to MDMel-WT cells, normalized to m36B4 (RplpO) as a reference gene ( $n=3$ for $\operatorname{lgfbp} 6, n=4$ for Angpt/4, $n=5$ for the rest). Bottom: Zymography to assess MMP-9 activity in the conditioned medium of MDMeI-WT and MDMel-KI cells (representative of $n=3$ ). (E) Concentration of CCL5 in the conditioned medium of MDMel-WT and MDMel-KI cells $(n=3)$. (F) Percentage of transcripts in each polysomal fraction quantified by quantitative real-time PCR $(n=3)$. Multiple unpaired 2-tailed $t$ test. (C) Representative image showing PCR-amplified cDNA fragments of the indicated targets $(n=3)$. The loading control (m36B4) is the same as for the data in Figure 3F, as the samples were run in parallel. (H) Fold change of indicated mRNAs in siNgfr-2-transfected (see Supplemental Table 4) MDMel-WT cells relative to the control group, normalized to m36B4 $(R p / p O)(n=3)$. (I) Correlation of the expression of indicated genes with the expression of NGFR (HTSeq [https://xenabrowser.net/datapages/?dataset=TCGA-SKCM.htseq_fpkm.tsv\&host=https\%3A\%2F\%2Fgdc. xenahubs.net\&removeHub=https $\% 3 \mathrm{~A} \% 2 \mathrm{~F} \% 2 \mathrm{Fxena}$.treehouse.gi.ucsc. edu\%3A443], fragments per kilobase of transcript per million mapped reads [FPKM]) in Genomic Data Commons (CDC) TCCA Melanoma data set (SKCM cohort, $n=477$ ). Spearman rank-order. (C-E and $\mathbf{H}$ ) Two-sided unpaired $t$ test. (C) Data are represented as mean \pm SEM. (D-H) Values represent the mean \pm SD.

in vitro (Supplemental Figure 3, C and D). Notably, previous studies by our group showed that the MKNK1/2-knockdown HBL cells are less invasive compared with their shCtrl counterparts, similar to the phospho-eIF4E-deficient MDMel-KI phenotype (9).

The data presented thus far support a previously unappreciated role for phospho-eIF4E in regulating melanoma plasticity exhibited as dedifferentiation/phenotype switching. A wealth of data suggests that the cell surface receptor NGFR serves as a molecular switch to promote melanoma dedifferentiation (29, $30,32,33)$. We show that $\mathrm{eIF} 4 \mathrm{E}^{\mathrm{KI}}$ melanomas had decreased NGFR expression compared with the eIF4 $4 \mathrm{E}^{\mathrm{WT}}$ tumors (Figure $3 \mathrm{C})$. Consistent with previous findings $(29,33)$, the regions staining positive for NGFR were restricted to areas that were negative for Melan-A (Supplemental Figure 3E). Additionally, NGFR expression was also decreased in both MDMel-KI melanoma cell lines compared with the MDMel-WT cell lines (Figure 3D and Supplemental Figure 3F). Although the Ngfr mRNA levels were 3- to 5-fold lower in the MDMel-KI cells (Figure 3D and Supplemental Figure 3F), given the robust decrease of NGFR at the protein level and the crucial role of phospho-eIF4E in mRNA translation, we next determined whether NGFR expression could be under the translational control of phospho-eIF4E. We subjected our MDMel cell lines to polysome profiling, a technique used to separate efficiently translated mRNAs bound to heavy polysomes from poorly translated mRNAs in light polysomes (47). Representative polysome gradient profiles from MDMel-KI and MDMel-WT samples overlapped (Figure 3E), consistent with the role of phospho-eIF4E in regulating the translation of selective mRNAs, without altering global protein synthesis (7-10). Quantitative PCR analysis of RNA isolated from heavy- and light-polysome-bound fractions in MDMel-WT and MDMel-KI cells indicated that a lack of phospho-eIF4E led to a redistribution of Ngfr mRNAs from heavy (efficiently translated) to light (poorly translated) polysomes (Figure 3F). This is consistent with the notion that the phosphorylation of eIF4E bolsters the translation of Ngfr mRNA. Knockdown of Ngfr in the MDMel-WT cells significantly impaired cell invasion (Figure $3 G$ ), with no change in proliferation (Supplemental Figure 3, G and H). Furthermore, control over the regulation of NGFR expression, upstream of phospho-eIF4E, is MNK1/2 dependent. We show that pharmacologic inhibition of MNK1/2 using the small-molecule inhibitor SEL201 (9) decreased NGFR expression in MDMel-WT cells and inhibited their invasion (Figure $3 \mathrm{H}$ ), without impairing proliferation (Supplemental Figure 3I). We further validated our findings in human melanoma cell lines. Using previously prepared heavy- versus light-polysome fractions (9), we showed that the translation of NGFR was inhibited in MKNK1/2-silenced human HBL cells (Supplemental Figure 4A). Similarly, SEL201 decreased NGFR and increased MITF expression in HBL and the invasive human melanoma cell line A375 (Supplemental Figure 4B).

Given the importance of NGFR-driven plasticity in drug-resistant melanoma cells $(30,48)$, we next tested whether blocking MNK1/2 would reverse phenotype switching in cells with acquired resistance to BRAF inhibition. We modeled this in 2 proliferative BRAF-mutant melanoma cell lines, SK-Mel-28 and WM164, through chronic exposure to the BRAF inhibitor vemurafenib (30, 48). Compared with invasive A375 cells, the SK-Mel-28 and WM164 cell lines expressed lower levels of NGFR and phospho-eIF4E, and showed increased MITF expression (Supplemental Figure 4C). However, following the acquisition of resistance, the SK-Mel28R and WM164R vemurafenib-resistant cells proliferated more slowly and invaded better, along with increased expression of NGFR and phospho-eIF4E and decreased MITF level, compared with the parental cells (Supplemental Figure 4, D-F). Treatment with SEL201 reversed the BRAF inhibitor-induced phenotype switch observed in SK-Mel-28R and WM164R, concomitantly with decreased invasion, decreased NGFR, and increased MITF expression (Supplemental Figure 4, F and G). Finally, SEL201 significantly inhibited the growth of SK-Mel-28R and WM164R cells maintained in vemurafenib (Supplemental Figure 4H).

Together, our data indicate that repressing the MNK1/2eIF4E axis suppresses melanoma dedifferentiation and invasion by decreasing NGFR protein expression.

Inhibition of phospho-eIF4E blocks proinflammatory cytokine secretion. Inflammation promotes dedifferentiation in melanoma, which in turn leads to an altered cytokine/chemokine profile (28-30, 34). Thus, we next monitored the expression of cytokines and chemokines present in the conditioned media harvested from eIF $4 \mathrm{E}^{\mathrm{WT}}$ and $\mathrm{eIF} 4 \mathrm{E}^{\mathrm{KI}}$ melanomas (Figure $4 \mathrm{~A}$ ). A set of secreted factors that are known to promote cancer cell invasion were found to be decreased in the eIF $4 \mathrm{E}^{\mathrm{KI}}$ melanomas. These included angiopoietin-2 (ANGPT2), CCL2, CCL12, CCL5, IGFBP-2, IGFBP-6, IL-6, and MMP-9 (Figure 4B and Supplemental Figure 5A). Many of these factors (angiopoietins, CCL2, CCL12, IGFBP-2, IGFBP-6) have never been thoroughly investigated in melanoma phenotype switching, but are represented in large-scale RNA-Seq 

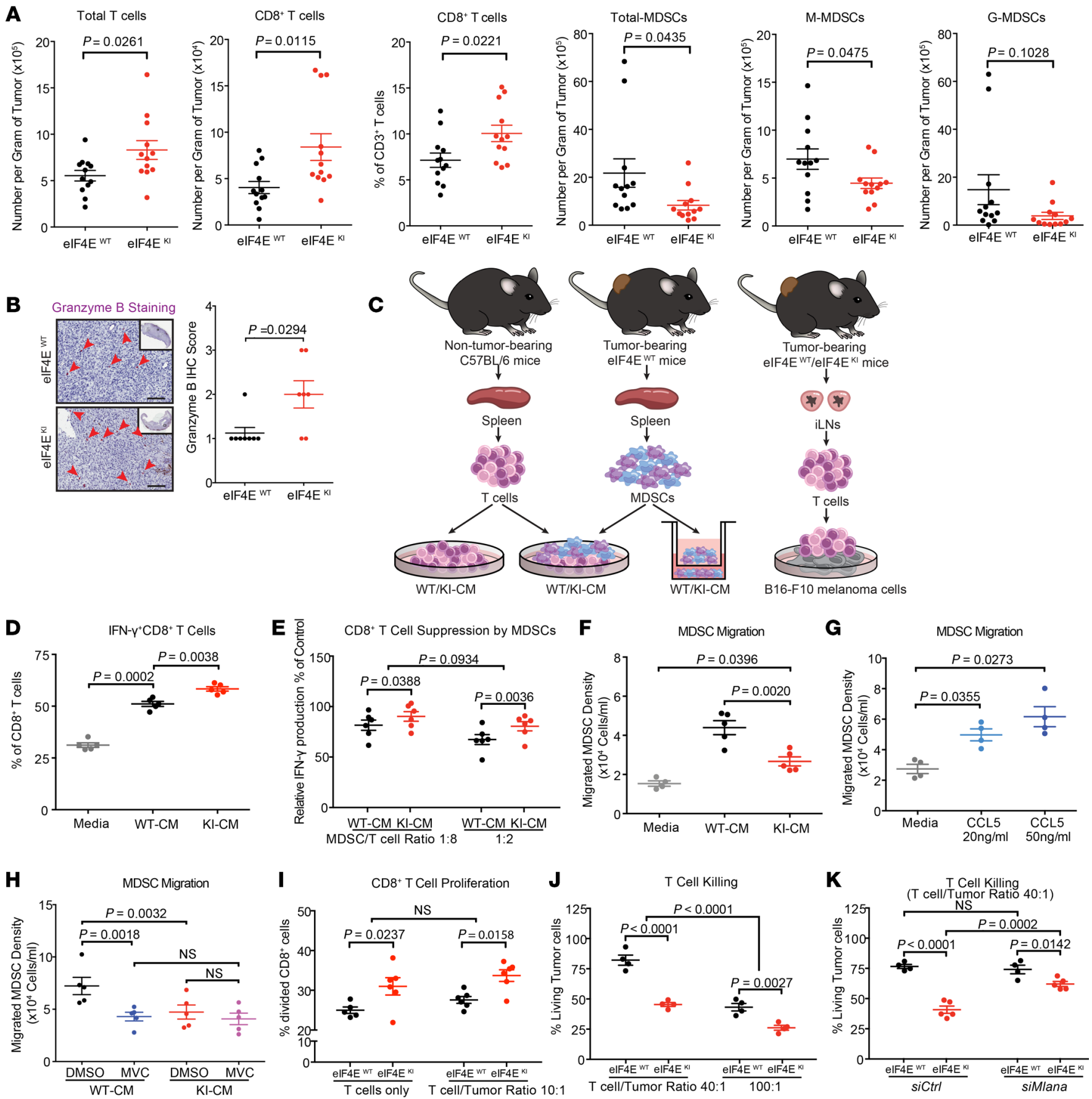

Figure 5. Phospho-elF4E deficiency impairs melanoma immunosuppression. (A) Immune cell populations infiltrated into the melanomas from $B R a f^{c A /+} /$ Pten $^{10 x / 10 x}$ elF4E ${ }^{\mathrm{WT}}$ and elF4E ${ }^{\mathrm{Kl}}$ mice (day 50). (B) Representative elF4E ${ }^{\mathrm{WT}}$ and elF4E ${ }^{\mathrm{Kl}}$ tumor samples (day 50) with IHC staining for granzyme B (left; scale bars: $100 \mu \mathrm{m}$ ) and corresponding scores (right). (C) Schematic of ex vivo experimental designs. (D) Percentage IFN- $\gamma$-producing CD8 ${ }^{+}$cells, stimulated and cultured in the conditioned medium from elF4E ${ }^{W T}$ or elF4E ${ }^{\mathrm{KI}}$ primary melanoma cultures (WT-CM, KI-CM) or regular medium for 72 hours. (E) MDSC inhibition of IFN- $\gamma$ production in $C D 8^{+}$T cells, cultured in WT-CM or KI-CM, relative to corresponding MDSC-free control group. (F-H) MDSC migration toward WT-CM, KI-CM, or regular medium (F); medium containing recombinant murine CCL5 (C); and WT-CM or KI-CM upon maraviroc (MVC; $100 \mathrm{nM}$ ) treatment (H). (I) Percent division of CD8 ${ }^{+} T$ cells isolated from the iLNs of elF4E ${ }^{\mathrm{WT}}$ and elF4E $\mathrm{E}^{\mathrm{Kl}}$ tumor-bearing mice, cultured alone or with B16-F10 melanoma cells. One data point was excluded (Grubbs' test). (J) Percent viability of B16-F10 cells cocultured with T cells from elF4E $E^{\mathrm{WT}}$ and elF4E $\mathrm{E}^{\mathrm{Kl}}$ tumor-bearing animals. See also Supplemental Figure 6I. (K) Percent viability of B16-F10 cells, silenced or not for Melan-A (siMlana-1; see Supplemental Table 4), cocultured with T cells from elF4E $\mathrm{E}^{\mathrm{WT}}$ and elF4E ${ }^{\mathrm{Kl}}$ tumor-bearing animals, relative to corresponding control groups. Number of biological replicates (mice) is indicated in each graph. For ex vivo assays (C-K), all tumor-bearing mice were sacrificed between days 35 and 38. (A and B) Two-sided unpaired $t$ test. (D, F, and $\mathbf{G})$ One-way repeated-measures (RM) ANOVA with Tukey's test. (E and $\mathbf{H}-\mathbf{K})$ Two-way RM ANOVA with Šidák correction. All values are represented as mean \pm SEM. 
analyses of genes associated with this process $(45,49,50)$. Moreover, among these cytokines, many are linked with the expansion (such as IL-6), recruitment (such as CCL2, CCL12, and CCL5), and function (such as MMP-9) of immunosuppressive cells, such as myeloid-derived suppressor cells (MDSCs) (51-53). Given the importance of CCL5 and IL-6 in inflammation and immune suppression, we used ELISA to verify that these factors were significantly repressed in the supernatants harvested from the eIF $4 \mathrm{E}^{\mathrm{KI}}$ melanomas compared with the eIF4 $\mathrm{E}^{\mathrm{WT}}$ melanomas (Figure 4C).

We next sought to uncover the molecular mechanisms underlying the differential expression of soluble factors from the eIF$4 \mathrm{E}^{\mathrm{WT}}$ and eIF $4 \mathrm{E}^{\mathrm{KI}}$ melanomas. First, we used our murine-derived melanoma cell lines, devoid or not of phospho-eIF4E, to interrogate the cellular source of the differentially regulated factors. While the expression of most of these factors was decreased at the mRNA level (Figure 4D and Supplemental Figure 5B), CCL5 was decreased only at the protein level in the MDMel-KI cells, with no significant change in Ccl5 mRNA in comparison with the MDMelWT cells (Figure 4, D and E). Similar to our results in MDMel cell lines, mouse embryonic fibroblasts (MEFs) devoid of phospho-eIF4E (KI-MEFs) (7) expressed less CCL5 protein by ELISA (Supplemental Figure 5C), although Ccl5 mRNA levels remained unchanged in comparison with WT-MEFs (Supplemental Figure $5 \mathrm{E})$. Analysis of $\mathrm{Ccl} 5 \mathrm{mRNAs}$ in the polysomal fractions isolated from the MDMel-KI cells showed a significant shift of Ccl5 mRNAs from heavy- to light-polysome fractions (Figure 4, F and G). Similar polysome-profiling results revealed that the $C c l 5 \mathrm{mRNAs}$ were less efficiently translated in KI-MEFs compared with WT-MEFs (Supplemental Figure 5, D and E). Our data thus demonstrate that Ccl5 mRNA is a translational target of phospho-eIF4E.

As we have shown that phospho-eIF4E promoted melanoma phenotype switching via enhancement of the translation of $\mathrm{Ng} f \mathrm{r}$, we next asked whether the expression of the phospho-eIF4E-regulated cytokines/chemokines was also dependent on the expression of NGFR. To test this, we knocked down Ngfr in MDMel-WT cells using siRNA (siNgfr-2; Figure 3G and Supplemental Figure 5F). The changes in cytokine/chemokine mRNA profile following Ngfr knockdown resembled those of the MDMel-KI cells. Such changes included the downregulation of Ccl2, Ccl12, Igfbp2, Mmp9, and an unexpected upregulation of Igfbp6 (Figure 4H). Angpt2 and Angptl4 remained unchanged, indicating that the expression of these two genes may not be directly regulated through NGFR. Additionally, the phospho-eIF4E-regulated cytokine/chemokine gene signature (ANGPT2, ANGPTL4, CCL2, IGFBP2, IGFBP6, IL6, and MMP9) was positively correlated with NGFR expression in the human data set of The Cancer Genome Atlas (TCGA) (Figure 4I). Finally, SEL201 treatment of MDMel-WT cells decreased CCL5 protein level (Supplemental Figure 5G) and faithfully recapitulated several of the profound changes in the cytokine/chemokine mRNA profile that we observed in the MDMel-KI cells (Supplemental Figure 5H).

Phospho-eIF4E supports an immunosuppressive tumor microenvironment. eIF4E $\mathrm{E}^{\mathrm{KI}}$ melanomas show evidence of resisting phenotype switching, with (a) decreased NGFR expression, (b) repressed production of proinflammatory secreted factors, and (c) maintenance of Melan-A antigen expression, leading us to predict that suppressing phospho-eIF4E favors antitumor immunity. $\mathrm{BRAF}^{\mathrm{V} 600 \mathrm{E}} / \mathrm{PTEN}^{\text {null/null }}$ murine melanomas have been character- ized by the presence of immunosuppressive MDSCs and modest T cell infiltration $(31,54)$. Thus, this model can be used to assess the impact of melanomas lacking phospho-eIF4E on tumor immune cell infiltration. Immune phenotyping of eIF $4 \mathrm{E}^{\mathrm{WT}}$ and $\mathrm{eIF} 4 \mathrm{E}^{\mathrm{KI}}$ melanomas showed that the phospho-eIF4E-deficient melanomas were significantly more infiltrated with total $\mathrm{CD}^{+} \mathrm{T}$ cell and $\mathrm{CD}^{+}$cytotoxic $\mathrm{T}$ cell populations compared with eIF4E $\mathrm{E}^{\mathrm{WT}}$ melanomas (Figure 5A and Supplemental Figure 6, A-D). Importantly, the eIF4 $\mathrm{E}^{\mathrm{KI}}$ melanomas showed higher intratumoral granzyme B-positive cell infiltrates, a marker of cytolytic action (Figure 5B). Consistent with the proinflammatory cytokine signature associated with eIF $4 \mathrm{E}^{\mathrm{WT}}$ melanomas, these melanomas were more infiltrated with MDSCs compared with eIF4E $\mathrm{E}^{\mathrm{KI}}$ melanomas (Figure 5A and Supplemental Figure 6C). There were significantly fewer monocytic MDSCs (M-MDSCs) in the eIF4E $\mathrm{E}^{\mathrm{KI}}$ melanomas compared with their control counterparts (Figure 5A and Supplemental Figure 6C). Tumor outgrowth in our model was negatively correlated with the number of tumor-infiltrating $\mathrm{T}$ cells and positively correlated with MDSCs (Supplemental Figure 6C). No significant changes in dendritic cells or macrophages were found between the eIF $4 E^{\mathrm{WT}}$ and eIF $4 \mathrm{E}^{\mathrm{KI}}$ melanomas (Supplemental Figure 6E). Overall, these data suggest an antitumor immune effect in the melanomas where eIF4E cannot be phosphorylated.

We hypothesized that the augmented $\mathrm{CD} 8^{+} \mathrm{T}$ cell influx and diminished presence of MDSCs in eIF4 $4 \mathrm{E}^{\mathrm{KI}}$ melanomas might be functionally associated with the altered inflammatory signature identified in the conditioned medium harvested from the phospho-eIF4E-deficient melanomas (Figure 4B). To test this, we devised a number of ex vivo immune cell-based assays (Figure 5C). The ability of $\mathrm{CD}^{+} \mathrm{T}$ cells to produce IFN- $\gamma$ was enhanced in the presence of eIF4 $4 \mathrm{E}^{\mathrm{WT}}$ tumor-conditioned medium compared with normal culture medium (Figure 5D), an effect that was potentiated when conditioned medium obtained from the eIF $4 \mathrm{E}^{\mathrm{KI}}$ melanomas was used (Figure 5D). There was no differential effect on the proliferation of $\mathrm{CD}^{+} \mathrm{T}$ cells cultured in the conditioned medium from eIF $4 \mathrm{E}^{\mathrm{WT}}$ or eIF $4 \mathrm{E}^{\mathrm{KI}}$ melanomas (Supplemental Figure 6F). Moreover, tumor-conditioned medium also promoted the migration of MDSCs ex vivo (Figure 5F). However, the migration of MDSCs and their ability to suppress $\mathrm{CD} 8^{+} \mathrm{T}$ cell function were both impaired under culturing conditions containing $\mathrm{eIF} 4 \mathrm{E}^{\mathrm{KI}}$ melanoma-derived conditioned medium compared with eIF $4 \mathrm{E}^{\mathrm{WT}}$-conditioned medium (Figure 5, E and F, and Supplemental Figure 6G). CCL5 is well known to promote the migration of $\operatorname{MDSCs}(55,56)$. In our model, the addition of recombinant murine CCL5 to the culturing medium promoted MDSC migration, thus recapitulating the phenotype observed using eIF $4 \mathrm{E}^{\mathrm{WT}}$ tumor-conditioned medium (Figure 5, F and G). Conversely, the CCR5 antagonist maraviroc, which blocks the CCL5/CCR5 axis, significantly decreased MDSC migration toward the eIF $4 \mathrm{E}^{\mathrm{WT}}$-conditioned medium (Figure $5 \mathrm{H}$ ). Such an effect was not observed using the eIF4 $\mathrm{E}^{\mathrm{KI}}$-conditioned medium, which contained low CCL5 levels (Figure 5H). Together, these data suggest that the activation of the CCL5/CCR5 axis, regulated by phospho-eIF4E, is crucial for the recruitment and immunosuppressive function of MDSCs.

Additional important factors contributing to the lack of $\mathrm{T}$ cells in melanomas have been proposed, such as a low presence of tumor antigens, including Melan-A and GP100, in highly 
A
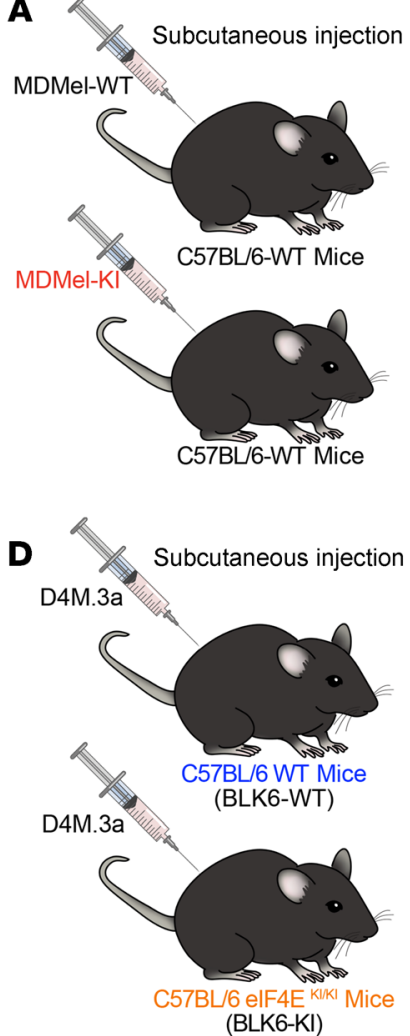

G

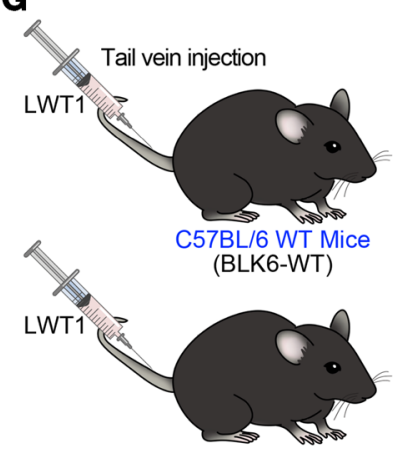

C57BL/6 elF4E KI/KI Mice

(BLK6-KI)
B

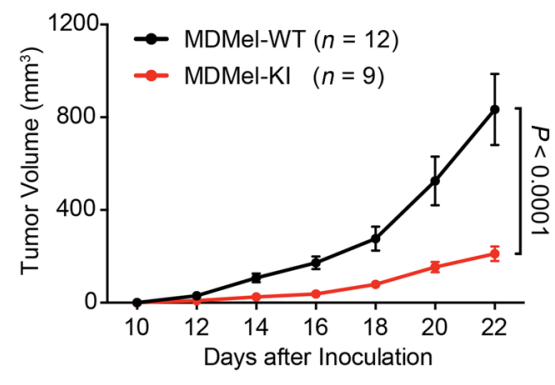

E

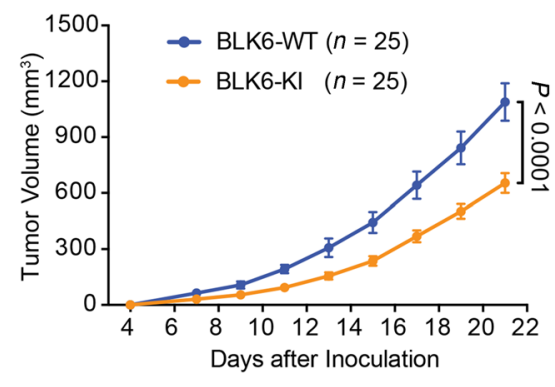

H
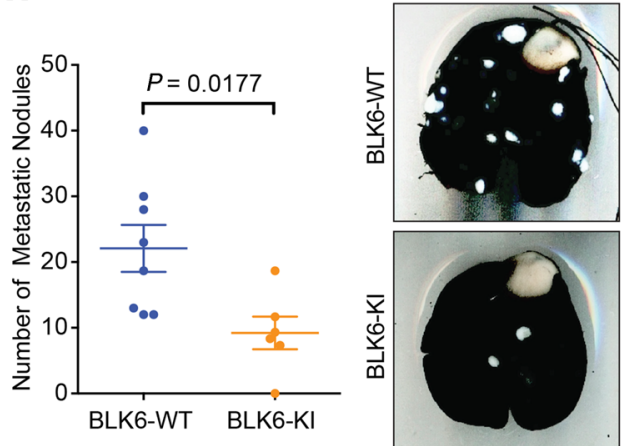

C
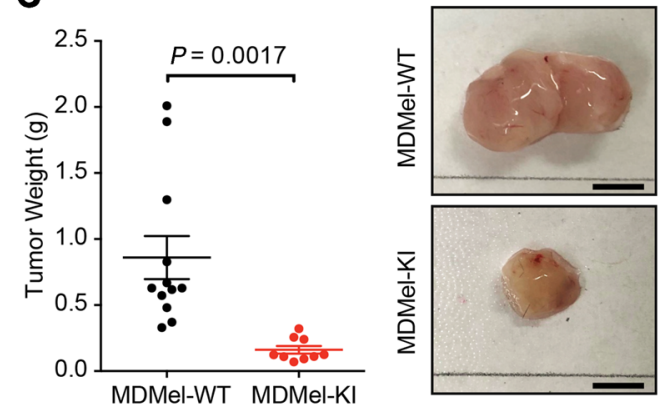

$\mathbf{F}$
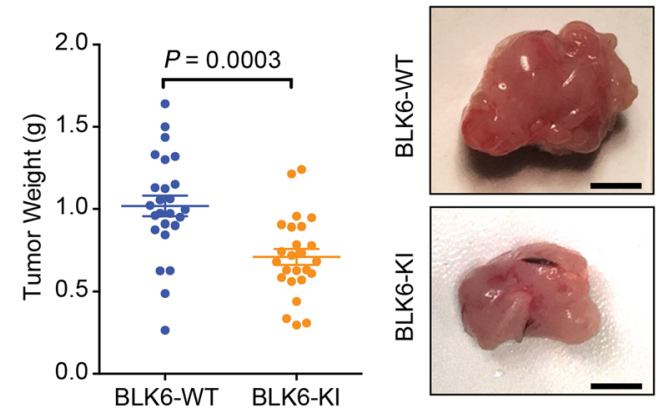

I
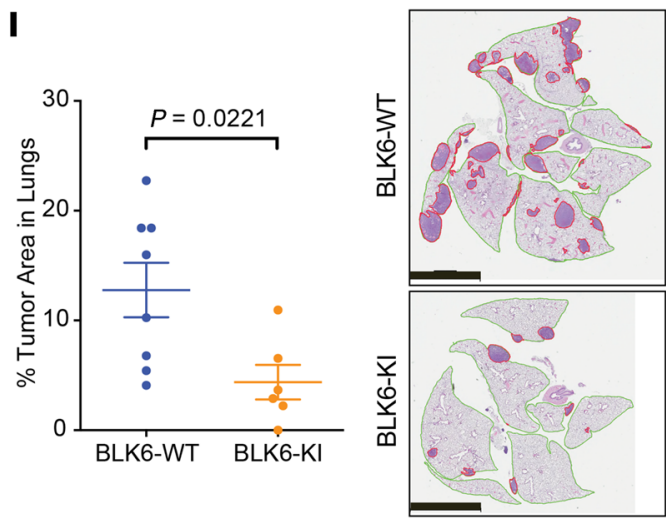

Figure 6. Tumor cell-intrinsic and -extrinsic phospho-elF4E facilitates melanoma outgrowth and metastasis. (A, D, and G) Schematic of the experimental design. (B and C) Growth curve (B) and tumor weight (C, left; day 22) with representative pictures (C, right; scale bars: $5 \mathrm{~mm}$ ) of MDMel-WT- and MDMel-KI-derived melanomas. (E and $\mathbf{F}$ ) Growth curve $(\mathbf{E})$ and tumor weight (F, left; day 21) with representative pictures (F, right; scale bars: $5 \mathrm{~mm})$ of D4M.3a-derived melanomas in BLK6-WT and BLK6-KI mice. (H and I) Number of metastatic nodules (H, left) with representative pictures of India Inkinflated lungs (H, right), and percentage of tumor area (I, left) with representative images of H\&E-stained lung sections (I, right; scale bars: $4 \mathrm{~mm}$ ), from BLK6-WT mice and BLK6-KI mice, after tail vein injection of LWT1 cells (day 21). (B and E) Two-way ANOVA. (C, F, H, and I) Two-sided unpaired $t$ test. All values are represented as mean \pm SEM.

dedifferentiated melanomas $(28,29)$. Therefore, we hypothesized that the increased presence of $\mathrm{CD} 8^{+} \mathrm{T}$ cells and granzyme $\mathrm{B}$-positive cells in the eIF $4 \mathrm{E}^{\mathrm{KI}}$ melanomas may be a consequence of the retention of melanocytic antigens in these tumors. To test this, we isolated $\mathrm{T}$ cells from the draining iLNs of eIF $4 \mathrm{E}^{\mathrm{WT}}$ or eIF $4 \mathrm{E}^{\mathrm{KI}}$ tumor-bearing mice at a time point when iLN metastasis was detectable in both mouse cohorts (Figure 5C, right). $\mathrm{CD} 8^{+}$ $\mathrm{T}$ cells isolated from the eIF4 $\mathrm{E}^{\mathrm{KI}}$ lymph nodes were more proliferative compared with $\mathrm{T}$ cells isolated from the eIF $4 \mathrm{E}^{\mathrm{WT}}$ lymph nodes, when cultured alone or with B16-F10 melanoma cells that express high levels of Melan-A and GP100 (Figure 5I and Supplemental Figure $6 \mathrm{H}$ ). Furthermore, using an ex vivo $\mathrm{CD}^{+}$cytotoxicity assay, we showed that B16-F10 melanoma cells were more effectively killed when cultured with phospho-eIF4E-deficient eIF4E ${ }^{\mathrm{KI}} \mathrm{CD} 8^{+} \mathrm{T}$ cells, compared with being cultured with WT $\mathrm{CD}^{+} \mathrm{T}$ cells (Figure $5 \mathrm{~J}$ and Supplemental Figure 6I). The eIF $4 \mathrm{E}^{\mathrm{KI}}$ $\mathrm{CD}^{+} \mathrm{T}$ cells were highly cytotoxic against the B16-F10 melanoma cells, as increasing the $\mathrm{T}$ cell/tumor cell ratio led to more 
A

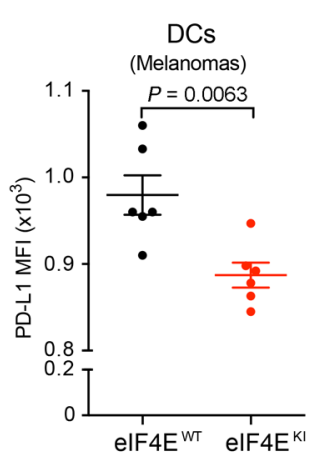

BRaf $^{C A /+} /$ Pten $^{\text {lox/lox }}$ Model

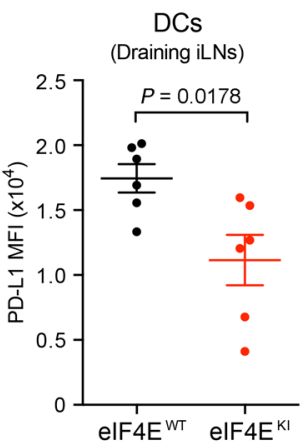

C

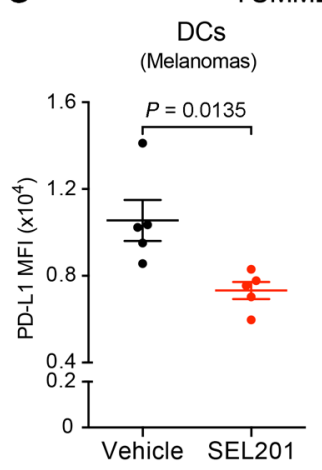

B
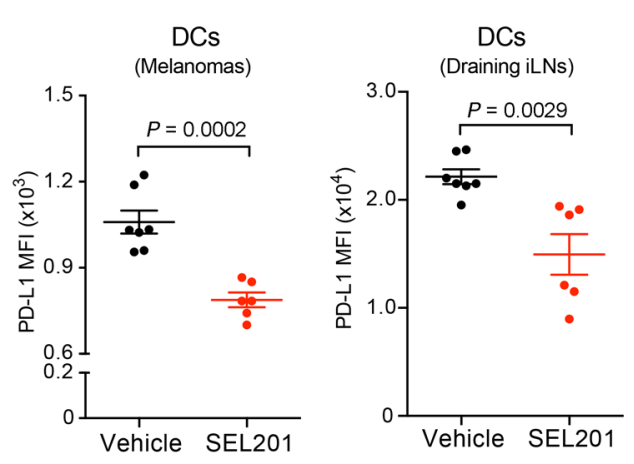

D

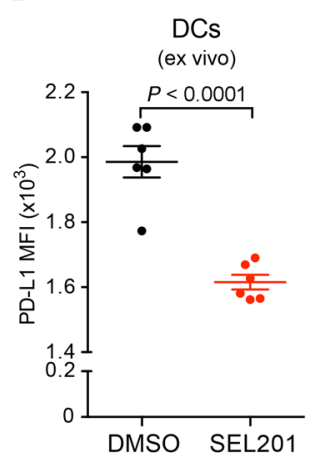

$\mathbf{F}$

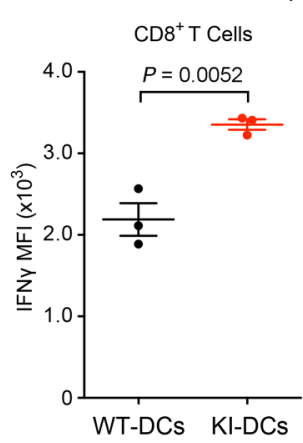

Figure 7. The MNK1/2-elF4E axis regulates PD-L1 expression on DCs. (A-C) PD-L1 expression on DCs in primary tumors (left) and draining iLNs (right) from $B R a f^{C A /+} /$ Pten $^{\text {lox/lox }}$ elF4E ${ }^{\mathrm{WT}}$ and elF4E ${ }^{\mathrm{KI}}$ mice $(\mathbf{A})$, vehicle- or SEL201-treated BRaf ${ }^{[A /+} /$ Pten $^{\text {lox/lox }}$ elF4E $\mathrm{E}^{\mathrm{WT}}$ mice (B), and vehicle- or SEL201-treated BLK6-WT mice bearing YUMMER1.7-derived melanomas (C). (D) PD-L1 expression on DCs isolated from BLK6-WT mice and cultured ex vivo with or without SEL201 for 18 hours. (E and $\mathbf{F}) \mathrm{T}$ cells were isolated from spleens of OT-1 mice and cocultured with OVA peptide-pulsed WT-DCs and KI-DCs for 72 hours. (E) Percentage CD25+CD44+ cells (left) and IFN- $\gamma^{+} \mathrm{CD} 44^{+}$cells (right) out of $\mathrm{CD} 8^{+} \mathrm{T}$ cells. (F) IFN- $\gamma$ expression on all $\mathrm{CD} 8^{+} \mathrm{T}$ cells (left) and IFN $-\gamma^{+} \mathrm{CD} 8^{+} T$ cells (right). See Supplemental Figure $8 \mathrm{~A}$ for detailed experimental design. Number of biological replicates is indicated in each graph. Two-sided unpaired $t$ test. All values are represented as mean \pm SEM.
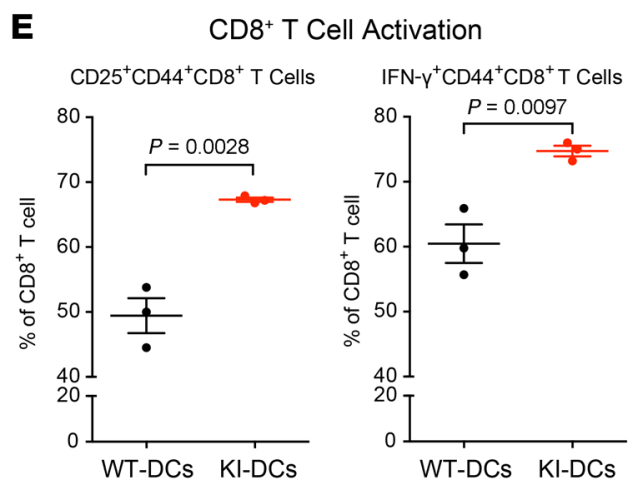

robust tumor cell killing, while $\mathrm{T}$ cells isolated from the lymph nodes of non-tumor-bearing mice showed minor tumor cell killing (Figure 5J and Supplemental Figure 6I). Finally, knockdown of Melan-A in B16-F10 cells significantly decreased the tumor cell killing ability of eIF $4 \mathrm{E}^{\mathrm{KI}} \mathrm{CD}^{+} \mathrm{T}$ cells (Figure $5 \mathrm{~K}$ and Supplemental Figure 6, J and K). These data suggest that the retention of Melan-A expression in eIF $4 \mathrm{E}^{\mathrm{KI}}$ melanomas leads to their recognition and eradication by $\mathrm{CD} 8^{+} \mathrm{T}$ cells.

Tumor cell-extrinsic phospho-eIF4E regulates melanoma outgrowth, metastasis, and PD-L1 expression on dendritic cells and MDSCs. In our BRaf $\mathrm{CA} /+^{-} / \mathrm{Pten}^{\text {lox/lox }}$ eIF $4 \mathrm{E}^{\mathrm{KI}}$ autochthonous mouse model of melanoma (Figure 1A), phospho-eIF4E is depleted in both the melanoma cells and the cells that constitute the tumor microenvironment (TME). To uncouple potential tumor-intrinsic and -extrinsic effects of phospho-eIF4E on melanoma outgrowth, we used the MDMel melanoma cells (Figure 3A), which can be injected into syngeneic immune-competent C57BL/6 mice (Fig- ure 6A). MDMel-KI-derived melanomas, where only the tumor cells are devoid of phospho-eIF4E, showed a significant defect in tumor outgrowth compared with MDMel-WT-derived melanomas (Figure 6, B and C).

Our observation that phospho-eIF4E-deficient $\mathrm{CD}^{+} \mathrm{T}$ cells isolated from the eIF4 $\mathrm{E}^{\mathrm{KI}}$ lymph nodes proliferated better than their eIF4E $\mathrm{E}^{\mathrm{WT}}$ counterparts (Figure 5I) hinted that phospho-eIF4E in nontumor cells may also contribute to melanoma outgrowth and metastasis. We therefore injected phospho-eIF4E-expressing D4M.3a and YUMMER1.7 melanoma cells, both derived from the BRaf ${ }^{C A /+} /$ Pten $^{\text {lox/lox }}$ (eIF4E ${ }^{\mathrm{WT}}$ ) model $(41,57)$, subcutaneously into syngeneic C57BL/6 (BLK6-WT) mice or phospho-eIF4E-deficient C57BL/6 eIF4E $\mathrm{E}^{\mathrm{S209A} / \mathrm{S} 209 \mathrm{~A}}$ (BLK6-KI) mice (Figure 6D and Supplemental Figure 7A). Both D4M.3a and YUMMER1.7 cells grew more slowly in BLK6-KI mice lacking phospho-eIF4E compared with the same cells grown in BLK6-WT mice (Figure 6, D-F, and Supplemental Figure 7, A-C). Moreover, in an experimental 

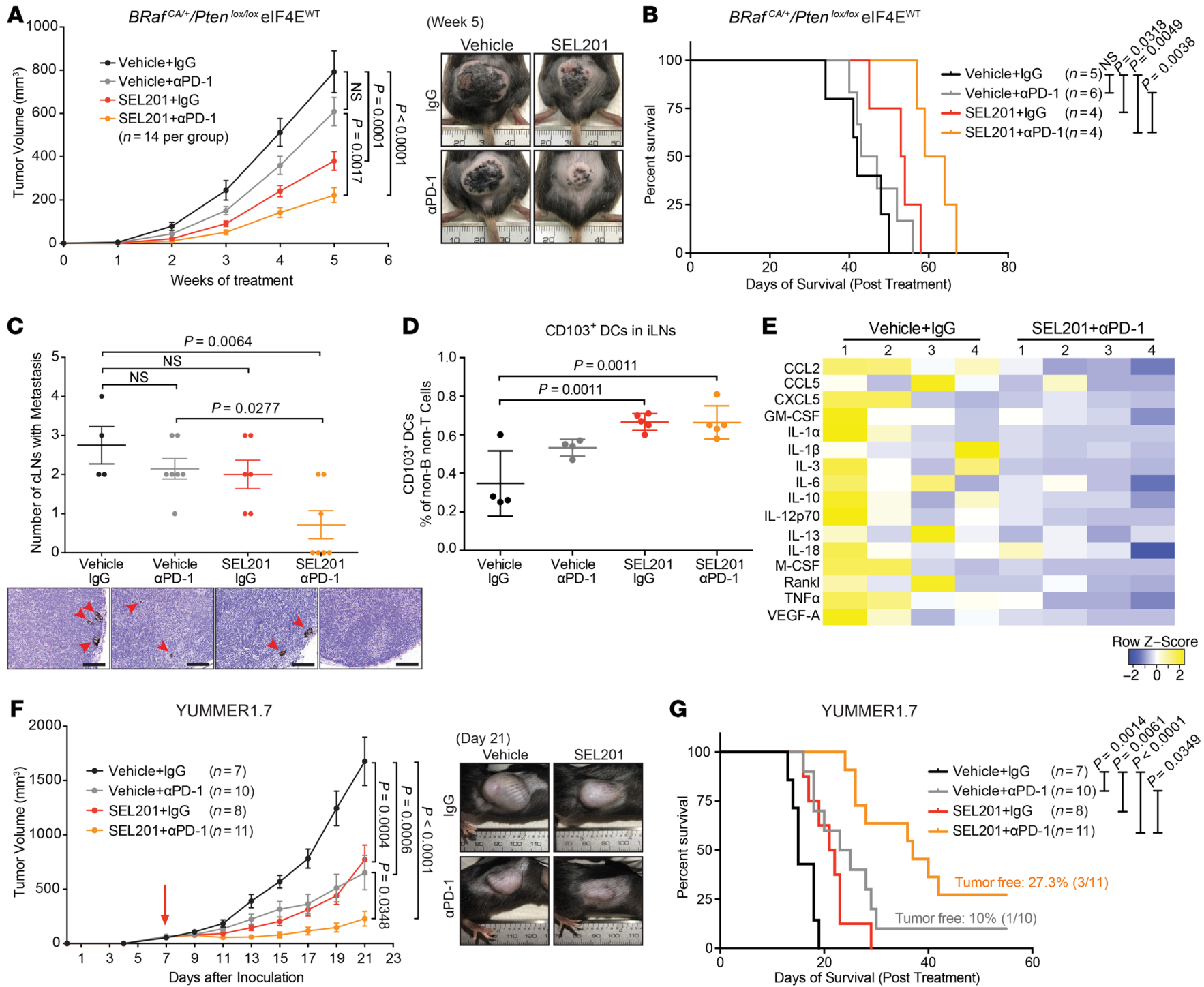

Figure 8. MNK1/2 inhibitor sensitizes melanoma to anti-PD-1 immunotherapy. (A) Growth curve (left) and representative pictures (right, week 5) of melanomas in elF4E ${ }^{W T}$ mice given control (Vehicle+lgG), monotherapies (Vehicle $+\alpha P D-1$, SEL201+lgG), or combination therapy (SEL201+ $\alpha$ PD-1). (B) Kaplan-Meier curves showing overall survival of mice in each group. (C) Number of metastasis-positive cLNs per mouse with representative images of H\&E-stained cLNs (scale bars: $100 \mu \mathrm{m}$ ) is presented for each group. (D) Percentage of CD103+ DCs out of non-B non-T cells in the iLNs from animals in each indicated group. (E) Relative plasma cytokine and chemokine levels in the control and combination therapy groups, detected by the MACPIX multiplexing system (Thermo Fisher Scientific). (F and $\mathbf{G})$ Melanoma growth (F, left), representative pictures (F, right; day 21), and Kaplan-Meier curves showing overall survival (G) of BLK6-WT mice bearing YUMMER1.7-derived melanoma, given the indicated treatments. (C-E) Mice were sacrificed after indicated treatment for 5 weeks. (F and $\mathbf{G}$ ) See individual tumor growth in Supplemental Figure 11A. Number of biological replicates (mice) is indicated in each graph. (A and F) Two-way RM ANOVA with Tukey's test. (C and D) Two-way ANOVA with Tukey's test. (B and G) Log-rank test. All values represent the mean \pm SEM.

model of lung metastasis (58), when we injected $\mathrm{BRAF}^{\mathrm{V} 600 \mathrm{E}}$-driven LWT1 murine melanoma cells into the tail vein of BLK6-WT mice or BLK6-KI mice, tumor burden was significantly decreased in the lungs of phospho-eIF4E-deficient mice (Figure 6, G-I).

From a therapeutic point of view, it is important to understand the impact of blocking the MNK1/2-eIF4E axis in nontumor cells of the immune microenvironment. A critical immune checkpoint receptor, PD-L1, has been reported to be under translational control in cancer cells $(59,60)$. Moreover, recent studies have demonstrated that PD-L1 expressed by dendritic cells (DCs) and MDSCs supports evasion from antitumor immunity (61-64).
We thus tested whether suppression of the MNK1/2-eIF4E axis blocks the expression of PD-L1 on DCs and MDSCs (Supplemental Figure 8A). Immune phenotyping revealed that phospho-eIF4E-deficient DCs, present in eIF4 $\mathrm{E}^{\mathrm{KI}}$ melanomas or eIF$4 \mathrm{E}^{\mathrm{KI}}$ draining lymph nodes, expressed less PD-L1 compared with their eIF4E $\mathrm{E}^{\mathrm{WT}}$ counterparts (Figure 7A). Using 2 different mouse models, we showed that DCs present in the melanomas and draining lymph nodes of mice treated with the MNK1/2 inhibitor SEL201 also expressed less PD-L1 (Figure 7, B and C). Moreover, ex vivo SEL201 treatment decreased PD-L1 expression on freshly extracted DCs (Figure 7D). Consistent with recent discoveries 
A

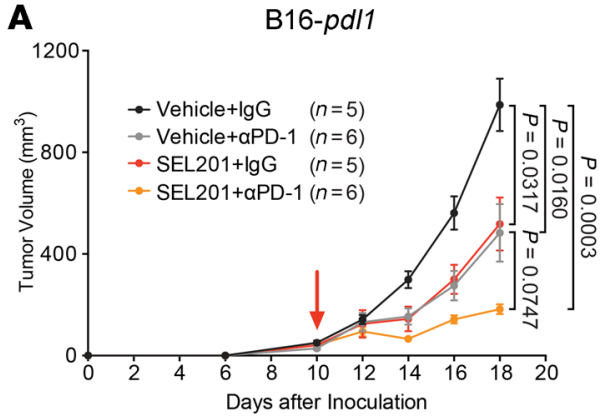

C
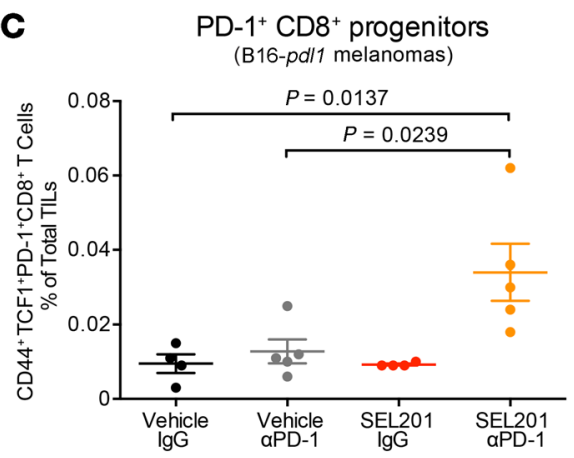

E

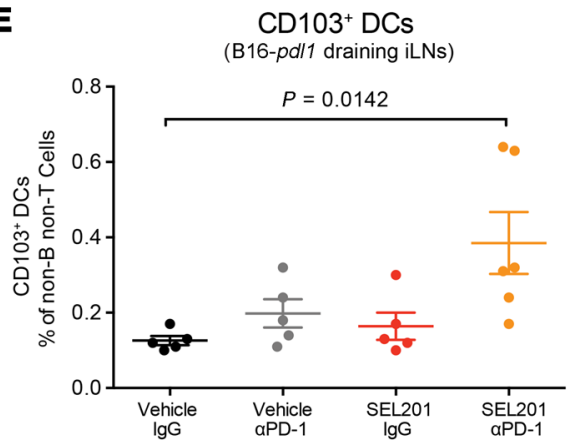

(Day 18)

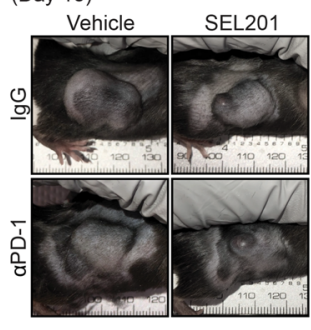

B

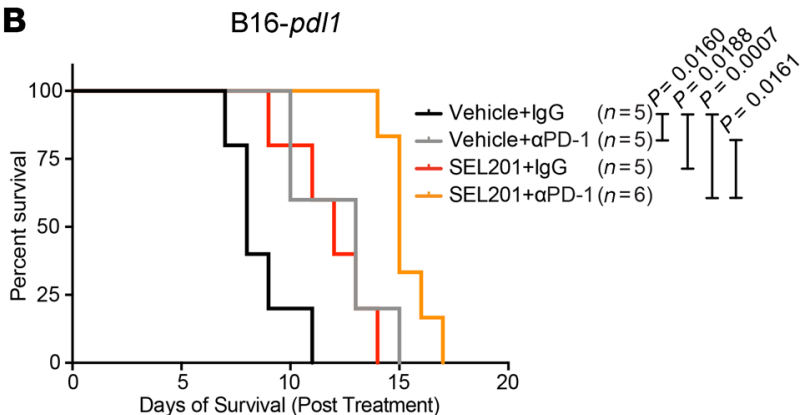

D
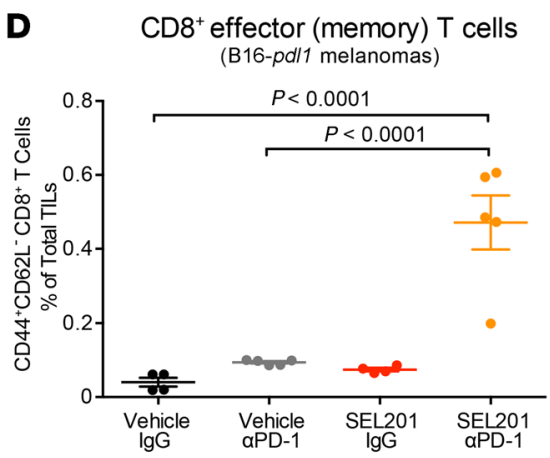

$\mathrm{CD} 103^{+} \mathrm{DCs}$

(B16-pdl1 melanomas)

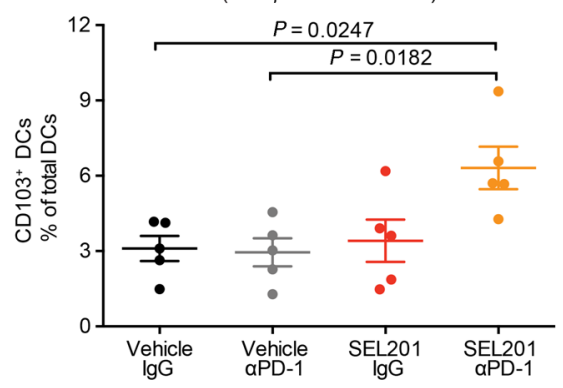

$\mathrm{CD}^{+}$central memory $\mathrm{T}$ cells (B16-pdl1 melanomas)

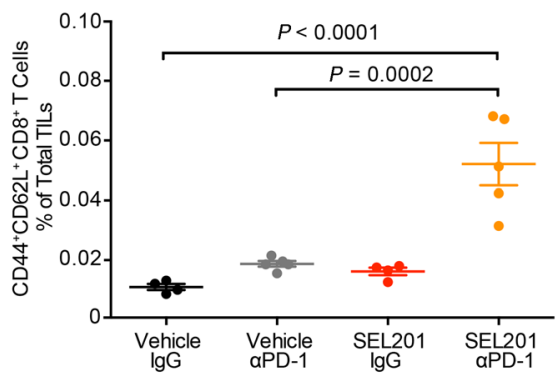$$
\text { . }
$$

Figure 9. Combination of MNK1/2 inhibitor and anti-PD-1 immunotherapy increases specific intratumoral T cell subsets. (A and B) Melanoma growth (A, left), representative pictures (A, right; day 18), and Kaplan-Meier curves showing overall survival (B) of BLK6-WT mice bearing B16-pdI1-derived melanoma, given the indicated treatments. (C-E) Abundance of indicated immune cell populations in B16-pdl1-derived melanomas or draining iLNs of each treatment group. Mice were sacrificed at endpoint, indicated in B, with 1 mouse (Vehicle+ $\alpha$ PD-1) removed from panel B because of a non-tumor-related death (see Supplemental Figure 11B). TIL, tumor-infiltrating lymphocyte. (A and B) See individual tumor growth in Supplemental Figure 11B. Number of biological replicates (mice) is indicated in each graph. (A) Two-way RM ANOVA with Tukey's test. (B) Log-rank test. (C-E) Two-way ANOVA with Tukey's test. All values represent the mean \pm SEM.

that blocking PD-L1 on DCs promotes T cell activation $(61,62)$, we showed that $\mathrm{CD} 8^{+} \mathrm{T}$ cells cocultured with phospho-eIF4Edeficient DCs (KI-DCs) were more activated (Figure 7E) and produced more IFN- $\gamma$ (Figure 7F) compared with those cocultured with WT-DCs. Similarly, the PD-L1 expression on MDSCs was also significantly decreased by genetic and pharmacologic inhibition of phospho-eIF4E (Supplemental Figure 8, B-D). As expected, along with decreased PD-L1 expression, blocking the MNK1/2-eIF4E axis in MDSCs impaired their ability to suppress $\mathrm{CD}^{+} \mathrm{T}$ cell proliferation (Supplemental Figure 8, E and F).

Together, these data suggest that the phosphorylation of eIF4E both in melanoma cells and in specific immune cells within the TME facilitates melanoma outgrowth and metastasis. Thus, the systemic inhibition of phospho-eIF4E, using a MNK1/2 inhibitor, could be therapeutically beneficial for patients with melanoma.
Inhibition of MNK1/2-mediated IF4E phosphorylation sensitizes melanoma to immune checkpoint blockade, with increased intratumoral stem-like $T C F 1^{+} P D-1^{+} C D 8^{+} T$ cells. Based on the data presented thus far, we propose that the protection of the eIF $4 \mathrm{E}^{\mathrm{KI}}$ mice against melanoma was likely a combined effect of phospho-eIF4E deficiency in the melanoma cells, and also in the cells of the TME. Inadequate tumor $\mathrm{T}$ cell influx, increased expression of $\mathrm{T}$ cell exhaustion markers, elevated PD-L1 levels on DCs, MDSC tumor infiltration, phenotype plasticity, and dedifferentiation can all underpin resistance to immunotherapies such as anti-PD-1 (19, 64-66). Our data support the concept that inhibiting the phosphorylation of eIF4E reverses melanoma plasticity, increases $\mathrm{CD}^{+}$TILs, and decreases PD-L1 expression on DCs. We thus suggest a promising strategy to block phospho-eIF4E, using MNK1/2 inhibitors, to augment responses to immune checkpoint inhibitors (ICIs). 
To test this, BRafCA/+ $/$ Pten $^{l o x} /$ lox $\left(\mathrm{eIF} 4 \mathrm{E}^{\mathrm{WT}}\right.$ ) melanomas were initiated, and when the melanomas were first visible, the mice were given SEL201 alone or in combination with an anti-PD-1 monoclonal antibody. Anti-PD-1 monotherapy alone did not significantly affect macroscopic melanoma outgrowth, metastasis, and the survival of animals (Figure 8, A-C, and Supplemental Figure 9, $\mathrm{A}-\mathrm{C})$, consistent with prior studies showing that this mouse model is insensitive to ICIs $(54,67)$. In contrast, SEL201 reduced both primary melanoma outgrowth and distant lymph node metastasis (Figure 8, A and C) and improved the survival of animals (Figure 8B). SEL201 administration in mice demonstrated robust circulating levels of drug (Supplemental Figure 9D) and on-target engagement, as shown by the repression of phospho-eIF4E expression in the melanomas (Supplemental Figure 9B). These data recapitulate ourobservationsinthephospho-eIF4E-deficient BRafCA/+ $/ \mathrm{Pten}^{\text {lox } / \text { lox }}$ $\left(\mathrm{eIF} 4 \mathrm{E}^{\mathrm{KI}}\right)$ mice, and further support the therapeutic benefits of blocking the MNK1/2-eIF4E axis (9, 59, 68-71). Importantly, the combined administration of SEL201 and anti-PD-1 monoclonal antibody significantly reduced primary melanoma growth and local and distant lymph node metastasis and increased the overall survival of tumor-bearing $B R a f^{C A /+} / \mathrm{Pten}^{\text {lox/lox }}$ mice compared with either of the therapies alone (Figure $8, \mathrm{~A}^{-} \mathrm{C}$, and Supplemental Figure 9, A-C), without causing any overt toxicity (Supplemental Figure 9B). Moreover, in mice treated with SEL201 plus anti-PD-1, we observed increased $\mathrm{CD}_{103^{+}}$DCs in the draining lymph nodes (Figure 8D). $\mathrm{CD} 103^{+}$DCs prime tumor-specific $\mathrm{CD}^{+} \mathrm{T}$ cells by transporting antigens to regional lymph nodes, and are thus required for ICI-mediated antitumoral immune responses $(72,73)$.

Analysis of plasma cytokine and chemokine levels revealed that the robust antitumor effect of SEL201 combined with anti-PD-1 therapy was associated with enhanced peripheral antitumor immunity, characterized by a decrease in the immunosuppressive cytokine/chemokine signature, including IL-6, CCL2, and CCL5 (Figure $8 \mathrm{E})$. Such a peripheral cytokine/chemokine signature might have prognostic value to predict responders to combination therapy with MNK1/2 inhibitors and immune checkpoint blockade. Although demonstrating a better antitumor response, the combination therapy led to a profound decrease in a subset of circulating cytokines that have been previously associated with immune-related adverse events due to immune checkpoint blockade (Supplemental Figure $9 \mathrm{E}$ and ref. 74). Furthermore, in contrast to the BRafCA/+$/ \mathrm{Pten}^{\text {lox } / \text { lox }}$ eIF4E ${ }^{\mathrm{WT}}$ mice, the $B R a f^{\mathrm{CA} /+} / \mathrm{Pten}^{\text {lox/lox }}$ eIF $4 \mathrm{E}^{\mathrm{KI}}$ mice were sensitive to anti-PD-1 monotherapy, showing a marked inhibition in macroscopic tumor outgrowth (Supplemental Figure 9F).

Having shown improved outcomes in $\mathrm{BRafCA/+} / \mathrm{Pten}^{\text {lox } / \text { lox }}$ mice treated with combined MNK1/2 inhibitor and anti-PD-1 therapy, we next tested our combination in 2 melanoma models reported to be more ICI responsive. We hypothesized that the efficacy of the MNK1/2 inhibitor plus anti-PD-1 combination therapy would be even more robust using a more immunogenic mouse model, and effective regardless of the genomic classification of melanoma. The YUMMER1.7 melanoma model is highly immunogenic and sensitive to anti-PD-1 monotherapy (57), with $10 \%$ of mice showing durable antitumor responses (Figure 8, F and G). Compared with the anti-PD-1 monotherapy, the combined effect of the MNK1/2 inhibitor SEL201 and anti-PD-1 reduced tumor growth by an additional $60 \%$ (Figure $8 \mathrm{~F}$ ), and improved overall surviv- al (median 24 days vs. 37 days), with durable antitumor effects observed in $27 \%$ of treated mice (Figure $8 \mathrm{G}$ ). Similarly, in the aggressive triple-WT B16-pdl1 melanoma model (75), we showed that combined MNK1/2 inhibition and anti-PD-1 resulted in augmented tumor control and significantly improved overall survival (Figure 9, A and B).

We next determined the impact of combined inhibition of MNK1/2 and anti-PD-1 therapy on T cell dysfunction in the TME, which remains a major clinical challenge. We focused on characterizing stem-like $\mathrm{TCF} 1^{+} \mathrm{PD}-1^{+} \mathrm{CD} 8^{+} \mathrm{T}$ cells, a recently identified population of immune cells that are required for maintaining durable ICI-mediated tumor control $(76,77)$. We observed a profound increase in intratumoral $\mathrm{TCF} 1^{+} \mathrm{PD}-1^{+} \mathrm{CD} 8^{+} \mathrm{T}$ cells in mice given SEL201 plus anti-PD-1 combination therapy compared with all other treatment arms (Figure 9C). Importantly, the tumor-infiltrating $\mathrm{CD}_{4} 4^{+} \mathrm{CD} 62 \mathrm{~L}^{-} \mathrm{CD} 8{ }^{+}$effector (and/or effector memory) $\mathrm{T}$ cells and $\mathrm{CD} 44^{+} \mathrm{CD} 62 \mathrm{~L}^{+} \mathrm{CD} 8^{+}$central memory $\mathrm{T}$ cells were also more abundant in the combination therapy group (Figure 9D). Consistent with our observations in $\mathrm{BRafCA/+} / \mathrm{Pten}^{\text {lox } / \text { lox }}$ treated mice (Figure 8D), CD103 ${ }^{+}$DCs increased in both tumors and draining lymph nodes following the combination therapy (Figure 9E). The results we obtained with SEL201 were recapitulated using the MNK1/2 inhibitor eFT508 (Supplemental Figure 10, A-E), which is in clinical trials (NCT03616834, NCT04261218, NCT04622007; ClinicalTrials.gov). Specifically, we observed a comparable increase in intratumoral $\mathrm{TCF} 1^{+} \mathrm{PD}-1^{+} \mathrm{CD} 8^{+} \mathrm{T}$ cells in mice given eFT508 plus anti-PD-1 combination therapy (Supplemental Figure 10C), thus strengthening the translational impact of these findings.

\section{Discussion}

Translational control, including the MNK1/2-eIF4E axis, has been implicated in promoting tumor cell plasticity across different tumor types (78). In this study, we show an integral role for the MNK1/2eIF4E axis in regulating melanoma plasticity, which is often associated with resistance to therapies (79). Our study specifically links the MNK1/2-eIF4E axis with immune escape of melanoma through phenotype switching. Mechanistically, both tumor-intrinsic and TME-mediated regulation of phenotype switching has been proposed (26-35). Here, we identified NGFR, a receptor that links intracellular signaling with extracellular factors in the TME, as a key downstream translational target of phospho-eIF4E that drives phenotype switching. Our results show that genetic and pharmacologic blockade of phospho-eIF4E downregulates the expression of the NGFR protein via a mechanism involving the repression of NGFR mRNA translation. Inhibition of phospho-eIF4E in melanoma cells led to increased/preserved expression of the melanocytic antigens Melan-A and GP100, decreased production of proinflammatory and immunosuppressive cytokines/chemokines, and reduced tumor infiltration of MDSCs. In our interrogation of the role of MNK1/2 and phospho-eIF4E in immune cells, we reported that blocking this axis decreased PD-L1 expression on DCs and MDSCs, resulting in augmented $\mathrm{CD}^{+} \mathrm{T}$ cell function. Overall, these changes resulted in the observed diminished melanoma outgrowth, reduced invasiveness and metastasis, and increased sensitivity to anti-PD-1 immunotherapy, which all resulted from repressed MNK1/2-eIF4E axis activity. 
Our study highlights a growing appreciation for the utility of inhibitors of mRNA translation, such as MNK1/2 inhibitors, as modulators of the immune system, which could be exploited for promoting durable antitumor immune responses when combined with immunotherapy. Although it has been reported that phospho-eIF4E and the eIF4F complex regulate the expression of PD-L1 on tumor cells $(59,60)$, it is now well appreciated that PD-L1 expression by immune cells is a critical determinant of antitumor immunity (6164). We showed that blocking the MNK1/2-eIF4E axis repressed the expression of PD-L1 on DCs and MDSCs, leading to enhanced CD8 ${ }^{+}$ $\mathrm{T}$ cell responses (Figure 7 and Supplemental Figure 8). We speculate that the loss of PD-L1 on DCs, in the presence of a MNK1/2 inhibitor, relieves B7.1 inhibition by PD-L1 binding in cis, thus enabling the MNK1/2 inhibitor-treated DCs to better activate $\mathrm{CD}^{+} \mathrm{T}$ cells. Curiously, prior work has shown that PD-L1 deletion specifically in DCs results in better tumor control and increased expression of immune checkpoint receptors such as PD- 1 on $\mathrm{CD}^{+} \mathrm{T}$ cells (61), indicating that they are antigen-experienced. Here, we found an increased presence of the $\mathrm{PD}-1$-expressing $\mathrm{TCF}^{+}$stem-like $\mathrm{CD}^{+} \mathrm{T}$ cells in melanomas that were treated with a MNK1/2 inhibitor combined with anti-PD-1. This specific population of $\mathrm{PD}-1^{+} \mathrm{CD} 8^{+} \mathrm{T}$ cells is critical for durable antitumor immunity $(76,77)$.

Given the importance of the melanocyte differentiation antigens (e.g., Melan-A) in melanoma immunogenicity and in favoring a good response to immune checkpoint inhibitors $(26,29,33,80)$, the relevance of combined blockade of phenotype switching and immune checkpoints in melanoma should not be neglected. For instance, increased expression of melanocytic antigens stimulates a vigorous $\mathrm{T}$ cell response, which in turn induces regression of melanoma metastases in patients $(29,39,40,80)$. Accordingly, the dedifferentiation of melanoma has been demonstrated as a major mechanism for the acquired resistance of melanomas to immunotherapies $(26,28,29)$. Indeed, our study shows that blocking phospho-eIF4E can reverse the dedifferentiation of melanoma, leading to increased expression of MITF and Melan-A (and GP100), which significantly enhanced the infiltration of $\mathrm{T}$ cells and their functions in tumor recognition and elimination. Intriguingly, phospho-eIF4E expression and Melan-A expression are inversely correlated in patient-derived samples, further demonstrating an association between the activation of the MNK1/2-eIF4E axis and the dedifferentiation (phenotype switching) of human melanoma. Further increasing the translational relevance of our work, using the TCGA SKCM (Skin Cutaneous Melanoma) cohort, we report that high MKNK1 expression in patient-derived melanomas is positively correlated with (a) an immune-escape gene signature composed of PDCD1, CTLA4, LAG3, HAVCR2, BTLA, CD160, CD244, TIGIT, KIR3DL2, and CD274 (Supplemental Figure 10F), and (b) a panel of genes reflecting MDSC infiltration (ITGAM, CD33, S1OOA9, CCR5, CD40, IL10, IL4R, IL1B, IL1O, CSF2, CD14, and FUT4) (Supplemental Figure $10 \mathrm{G}$ and refs. 51-53). Additionally, our interrogation of the TCGA melanoma data set revealed that NGFR expression correlates with the phospho-eIF4E-regulated cytokine/chemokine signature (Figure 4I), except for CCL5, which we reported to be under the translational control of phospho-eIF4E. We acknowledge that these observations are derived from bulk tumor data and may be confounded by the presence of immune cell infiltrates. Nevertheless, we suggest, based on the data presented in this study, that MNK1 may be part of a regulatory network to drive immune suppression in human melanoma. Our future in vivo studies will center on understanding how inhibition of MNK1/2 leads to a reshaping of the immune microenvironment in melanoma using state-of-the-art single-cell imaging techniques, such as imaging mass cytometry.

Our findings are aligned with prior work demonstrating that BRAF and/or MEK inhibitors synergize with immune checkpoint blockade in melanoma, via increased antigen expression, decreased proinflammatory cytokine/chemokine production, and improved $\mathrm{T}$ cell infiltration $(65,81-84)$. However, such combinations can result in a high rate $(273 \%)$ of grade $3 / 4$ treatment-related adverse events in patients with melanoma (84). As MNK1/2 inhibitors have reached clinical trials (NCT03616834, NCT03690141, NCT04261218, NCT03258398, NCT04622007), it will be important to assess whether targeting of MNK1/2, downstream of activated BRAF/MEK/ERK, is potentially better tolerated. Encouragingly, several peripheral cytokines recently shown to predict immune-related toxicity of immune checkpoint blockade (74) were repressed when $B R a f^{C A /+} / \mathrm{Pten}^{\text {lox/lox }}$ mice were treated with combined anti-PD-1 and SEL201. Moreover, phenotype switching is independent of melanoma genomic classification (45, 46), and we have shown that MNK1/2 inhibition, which reversed phenotype switching in melanoma cells with different mutations (Figures 2 and 3 and Supplemental Figures 3 and 4), sensitized melanomas to anti-PD-1 therapy in models of BRAF-mutant and non-BRAF-mutant melanoma (Figures 8 and 9 and Supplemental Figures 9 and 10). Notably, in BRAF-mutant melanomas treated with BRAF inhibitors, acquired resistance can develop through increased plasticity $(30,48)$, which we have also shown is reversed by the blockade of MNK1/2 (Supplemental Figure 4). Therefore, compared with inhibitors of BRAF and/or MEK, MNK1/2 inhibitors have the potential (a) to be well tolerated, (b) to demonstrate efficacy in patients with BRAF-mutant and non-BRAF-mutant melanoma when combined with immunotherapy, and (c) to be used after disease progression on BRAF inhibitors.

In summary, we provide evidence that MNK1/2 inhibitors show efficacy in preclinical melanoma models by targeting phospho-eIF4E-regulated phenotype switching, and by serving as potent immune modulators that are likely most therapeutically beneficial when combined with immune checkpoint blockade.

\section{Methods}

Mice. C57BL/6N mice were purchased from Charles River Laboratories. C57BL/6 Tyr::CreER/BRafCA/+ $/$ Pten $^{\text {lox } / l o x}\left(\mathrm{eIF}^{\mathrm{C}} \mathrm{E}^{\mathrm{WT}}\right.$ ) mice (36) and C57BL/6 eIF4E $\mathrm{E}^{\mathrm{S} 209 \mathrm{~A} / \mathrm{S209A}}$ mice (7) were generated in-house. The BRafCA/+ $/$ Pten $^{\text {lox/lox/ }}$ Eif4 $e^{\mathrm{S209A} / S 209 A}\left(\mathrm{eIF} 4 \mathrm{E}^{\mathrm{KI}}\right)$ mice were generated by crossing of C57BL/6 eIF$4 \mathrm{E}^{\mathrm{S} 209 \mathrm{~A} / \mathrm{S} 209 \mathrm{~A}}$ and C57BL/6 Tyr::CreER/BRaf ${ }^{\mathrm{CA} /+} /$ Pten $^{\text {lox } / \text { lox }}$ (eIF4E ${ }^{\mathrm{WT}}$ ) mice. Genotyping was as indicated in Supplemental Table 2.

Detailed descriptions of experiments involving eIF $4 \mathrm{E}^{\mathrm{WT}}$ and eIF$4 \mathrm{E}^{\mathrm{KI}}$ mice with 4-HT-induced melanoma, subcutaneous injection of D4M.3a, YUMMER1.7, and B16-pdl1 cells into C57BL/6 mice, and in vivo LWT1 metastases can be found in Supplemental Methods.

Cells and reagents. The McGill-Derived murine Melanoma (MDMel) cell lines MDMel-WT ${ }^{88}$, MDMel-WT ${ }^{73}$, MDMel-KI ${ }^{61}$, and MDMel-KI ${ }^{58}$ were generated similarly to murine melanoma cell line D4M.3a used in this study (41) (see Supplemental Methods). Unless 
otherwise specified, all MDMel-WT cells refer to the MDMel-WT ${ }^{73}$ cell line and all MDMel-KI cells refer to the MDMel-KI ${ }^{61}$ cell line. The human vemurafenib-resistant SK-Mel-28R and WM164R cells were generated by culturing of the parental SK-Mel-28 and WM164 cells, respectively, in elevated doses of vemurafenib (starting at $0.5 \mu \mathrm{M}$ ). SK-Mel-28R and WM164R cells were maintained with $2.5 \mu \mathrm{M}$ and 1 $\mu \mathrm{M}$ vemurafenib, respectively. The human HBL-shCtrl and HBL-shMKNK1/2 cell lines were generated as previously described (9). SEL2O1 was provided by Selvita SA. eFT508 was purchased from Selleckchem (S8275). Vemurafenib was obtained from Plexxikon. The anti-PD-1 monoclonal antibody (RMP1-14, BE0146) and IgG isotype control (2A3, BE0089) were purchased from Bio X Cell.

Polysome profiling. Polysome profiling was performed as previously described (9). Briefly, MDMel-WT ${ }^{73}$, MDMel-KI ${ }^{61}$, WT-MEF, and KI-MEF cells were serum-starved overnight and then serum-stimulated for 2 hours. Cells were treated with cycloheximide $(100 \mu \mathrm{g} / \mathrm{mL}) 5 \mathrm{~min}$ utes before harvesting, then washed in cold PBS containing $100 \mu \mathrm{g} /$ $\mathrm{mL}$ cycloheximide, followed by centrifugation for 5 minutes at $390 \mathrm{~g}$. Cell pellets were lysed in hypotonic buffer (5 mM Tris- $\mathrm{HCl}$ [pH 7.5], 2.5 $\mathrm{mM} \mathrm{MgCl}, 1.5 \mathrm{mM} \mathrm{KCl}$ ) and $1 \times$ protease inhibitor cocktail containing $1 \mathrm{mM}$ DTT and RNase inhibitor (100 U). Samples were kept on ice for 12 minutes, and centrifuged at 13,523g for 7 minutes. The supernatants were loaded onto $10 \%-50 \%$ sucrose density gradient and centrifuged at 260,110 $g$ for 2 hours at $4^{\circ} \mathrm{C}$. The polysomal fractions were monitored and collected using a Teledyne ISCO one-rack Foxy R1 fraction collector.

Immunohistochemistry and scoring. Human melanoma tissue samples were acquired from the Institut Jules Bordet, and written informed consent from all patients was obtained in accordance with the Declaration of Helsinki. Immunohistochemical staining of human patient samples was performed on a Ventana Discovery Benchmark XT, while staining of mouse samples was performed as previously described (9). Briefly, formalin-fixed, paraffin-embedded tumor sections were stained with indicated antibodies (Supplemental Table 1) at 1:50 dilution, followed by a standard fast red detection protocol. Hematoxylin-counterstained slides were mounted with coverslips. Staining intensity was determined by clinically certified pathologists. The intensity of phospho-eIF4E staining in the human melanomas (scores ranged from 0.5 to 5 ) was further divided into a p-eIF $4 \mathrm{E}^{\text {hi }}$ group (scores $>2.5$ ) and a p-eIF $4 \mathrm{E}^{\mathrm{lo}}$ group (scores 0.5-1). Additional melanoma sections from the p-eIF4 $\mathrm{E}^{\text {hi }}$ and $\mathrm{p}$-eIF4E${ }^{10}$ groups were stained for Melan-A. All pathologists were blinded to all clinical data and antibodies used for immunohistochemistry.

Tumor-conditioned media. eIF $4 \mathrm{E}^{\mathrm{WT}}$ and $\mathrm{eIF} 4 \mathrm{E}^{\mathrm{KI}}$ melanomas were resected at day 35 to 38 after $4-\mathrm{HT}$ administration. Tumors were minced and digested in collagenase A to obtain single-cell suspension and cultured in advanced DMEM/F12 containing 5\% FBS, $1 \times$ GlutaMAX, and 1× Antibiotic-Antimycotic (penicillin/streptomycin/ amphotericin B; Thermo Fisher Scientific) at approximately 3 to 4 million cells per $15-\mathrm{cm}$ dish overnight. Then cells were washed with PBS and changed to serum-free DMEM/F12 media (1× Antibiotic-Antimycotic) for 24 hours. Media were harvested and protein concentrations were adjusted based on Bradford measurement. These media were then used for cytokine array or ELISA or supplemented with 10\% FBS to make tumor-conditioned media for ex vivo immune cell assays.

Immunophenotyping. Tumors were mechanically minced and digested in $4 \mathrm{mg} / \mathrm{mL}$ collagenase A (Sigma-Aldrich) at $37^{\circ} \mathrm{C}$ for 1 hour, and subsequently strained through $70-\mu \mathrm{m}$ nylon cell strainers to obtain single-cell suspension. When indicated, tumor-infiltrating leukocytes were enriched using the Histopaque-1119 density gradient separation method (Sigma-Aldrich). Single cells were stained with the indicated antibodies as well as a live/dead discrimination dye (Supplemental Table 5). Data were subsequently acquired with a Fortessa flow cytometer (BD Biosciences).

$D C / T$ cell coculture assay. DCs were isolated from spleens of C57BL/6 mice or C57BL/6 eIF4E $\mathrm{E}^{\mathrm{S} 209 \mathrm{~A} / \mathrm{S2} 29 \mathrm{~A}}$ mice using the EasySep Mouse Pan-DC Enrichment Kit (STEMCELL Technologies). Ten thousand DCs were pulsed with $13 \mathrm{pM}$ of ovalbumin peptide (SIINFEKL) per well in 96-well plates. Six hours after stimulation, $5 \times 10^{4}$ sorted $\mathrm{CD}^{+} \mathrm{T}$ cells from OT-1 mice were added (1:5 ratio). On day 3, $\mathrm{T}$ cells were stimulated with phorbol 12-myristate 13 -acetate (PMA; 50 $\mathrm{ng} / \mathrm{mL}$ ) and ionomycin $(500 \mathrm{ng} / \mathrm{mL}$ ) for 4 hours, and T cells were then examined for cytokine production and expression of cell surface activation markers using flow cytometry.

MDSC/T cell coculture assay. MDSCs were isolated from spleens of tumor-bearing eIF4 $\mathrm{E}^{\mathrm{WT}}$ or eIF4 $\mathrm{E}^{\mathrm{KI}}$ mice using the EasySep Mouse MDSC $\left(\mathrm{CD}_{\left.11 \mathrm{~b}^{+} \mathrm{Gr} 1^{+}\right)}\right.$Isolation Kit (STEMCELL Technologies). MDSCs were seeded together with 75,000 $\mathrm{T}$ cells at indicated ratios in CD3-coated 96-well plates. Media used in this assay are indicated in Supplemental Methods (see "T cell activation" section).

T cell/tumor cell coculture assay. B16-F10 melanoma cells were seeded in 24-well plates at 2000 cells per well or in 96-well plates at 400 cells per well 1 day before $T$ cell extraction. T cells were extracted from inguinal lymph nodes of eIF $4 \mathrm{E}^{\mathrm{WT}}$ or eIF $4 \mathrm{E}^{\mathrm{KI}}$ tumor-bearing animals and were purified using the EasySep Mouse T Cell Isolation Kit (STEMCELL Technologies) and stained with $1.4 \mathrm{ng} / \mathrm{mL}$ CFSE. T cells were added to the tumor cells in ImmunoCult-XF T Cell Expansion Medium (STEMCELL Technologies) supplemented with $20 \mathrm{ng} / \mu \mathrm{L}$ IL-2 at indicated T cell/tumor cell ratios. Cells were then cocultured for another 72 hours. $\mathrm{T}$ cells in 24-well plates were harvested to access proliferation and IFN- $\gamma$ production. T cells and dead tumor cells in 96-well plates were washed off by PBS, and tumor cells were fixed with $4 \%$ PFA and stained with crystal violet. Percentage survival was accessed by OD value in comparison with tumor cells cultured alone.

Statistics. In vitro data were represented as mean \pm SD. In vivo and ex vivo data were represented as mean \pm SEM. GraphPad Prism software was used to determine statistical significance of differences. Figure legends specify the statistical analysis used and define error bars. $P$ values are indicated in the figures, and $P$ values less than 0.05 were considered significant.

Study approval. Animal experiments were conducted according to the regulations established by the Canadian Council of Animal Care, and protocols approved by the McGill University Animal Care and Use Committee (2015-7672). Acquisition and the IHC study on human melanomas were approved by the Central Ethics Committee of the Institut Jules Bordet (CE2023).

\section{Author contributions}

FH, SVDR, CER, and CMK designed research studies. FH, CG, $\mathrm{MB}, \mathrm{MEI}, \mathrm{BC}$, and MA conducted experiments. FH, CG, MB, JRS, MEI, BC, QG, AE, WY, DP, JS, YZ, MA, AG, MM, MM, JF, EK, AB, and NG acquired data. FH, CG, MB, MEI, BC, AE, MGG, MA, AG, and TR analyzed and interpreted data. FH, MB, HUS, NS, IT, WHM, and SVDR wrote, reviewed, and/or revised the manuscript. CG, DD, NS, CAP, FJ, and GEG provided administrative, technical, or material support. WHM and SVDR supervised the study. 


\section{Acknowledgments}

This research was funded by the Canadian Institutes of Health Research (CIHR) (grant PJT-162260 to SVDR and grants MOP142281 and PJT-156269 to WHM and SVDR) and the Canadian Cancer Society (grant 703811 to WHM). This work was also supported by the Terry Fox Research Institute-Montreal Cancer Consortium (TFRI; grant 1084 to SVDR, WHM, and CER) and the Rossy Cancer Network. Development of MNK1/2 inhibitors by Ryvu Therapeutics has been cofinanced by the National Centre for Research and Development, INNOTECH Program (INNOTECH-K1/HI1/I6/157438/NCBR/12). FH, QG, and WY were endowed by McGill Integrated Cancer Research Training Program graduate studentships. FH and QG were sponsored by McGill Faculty of Medicine graduate studentships. QG and $\mathrm{AB}$ were financed by a Cole Foundation PhD fellowship. BC was financed by CIHR doctoral fellowship CGS-D. We thank David Fisher (Harvard Medical School, Boston, Massachusetts, USA) for his donation of the MITF antibody. We thank Christian Young, Naciba Benlimame, Lilian Canetti, Valeria Narykina, Sathyen A. Prabhu, and Sylvain Roux for experimental advice and technical supports.

Address correspondence to: Sonia V. del Rincón, Lady Davis Institute, Jewish General Hospital, 3999 Côte-Sainte-Catherine, Room E-503, Montréal, Quebec, Canada H3T 1E2. Phone: 514.340.8222 ext. 22246; Email: sonia.delrincon@mcgill.ca.
1. Cancer Genome Atlas Network. Genomic classification of cutaneous melanoma. Cell. 2015;161(7):1681-1696.

2. Waskiewicz AJ, et al. Mitogen-activated protein kinases activate the serine/threonine kinases Mnk1 and Mnk2. EMBO J.1997;16(8):1909-1920.

3. Fukunaga R, Hunter T. MNK1, a new MAP kinase-activated protein kinase, isolated by a novel expression screening method for identifying protein kinase substrates. EMBO J. 1997;16(8):1921-1933.

4. Carter JH, et al. Phosphorylation of eIF4E serine 209 is associated with tumour progression and reduced survival in malignant melanoma. Br J Cancer. 2016;114(4):444-453.

5. Fan S, et al. Phosphorylated eukaryotic translation initiation factor 4 (eIF4E) is elevated in human cancer tissues. Cancer Biol Ther. 2009;8(15):1463-1469.

6. Hsieh AC, Ruggero D. Targeting eukaryotic translation initiation factor $4 \mathrm{E}$ (eIF4E) in cancer. Clin Cancer Res. 2010;16(20):4914-4920.

7. Furic L, et al. eIF4E phosphorylation promotes tumorigenesis and is associated with prostate cancer progression. Proc Natl Acad Sci U S A. 2010;107(32):14134-14139.

8. Robichaud N, et al. Phosphorylation of eIF4E promotes EMT and metastasis via translational control of SNAIL and MMP-3. Oncogene. 2015;34(16):2032-2042.

9. Zhan Y, et al. MNK1/2 inhibition limits oncogenicity and metastasis of KIT-mutant melanoma. J Clin Invest. 2017;127(11):4179-4192.

10. Wendel HG, et al. Dissecting eIF4E action in tumorigenesis. Genes Dev. 2007;21(24):3232-3237.

11. Topisirovic I, et al. Phosphorylation of the eukaryotic translation initiation factor eIF4E contributes to its transformation and mRNA transport activities. Cancer Res. 2004;64(23):8639-8642.

12. Hodi FS, et al. Improved survival with ipilimumab in patients with metastatic melanoma. $N$ Engl J Med. 2010;363(8):711-723.

13. Robert $\mathrm{C}$, et al. Ipilimumab plus dacarbazine for previously untreated metastatic melanoma. N Engl J Med. 2011;364(26):2517-2526.

14. Robert C, et al. Pembrolizumab versus ipilimumab in advanced melanoma. $\mathrm{NEnglJMed}$. 2015;372(26):2521-2532.

15. Robert C, et al. Nivolumab in previously untreated melanoma without BRAF mutation. $N$ Engl J Med. 2015;372(4):320-330.
16. Ribas A, Wolchok JD. Cancer immunotherapy using checkpoint blockade. Science. 2018;359(6382):1350-1355.

17. Michot JM, et al. Immune-related adverse events with immune checkpoint blockade: a comprehensive review. Eur J Cancer. 2016;54:139-148.

18. Guo Q, et al. Translation of cancer immunotherapy from the bench to the bedside. Adv Cancer Res. 2019;143:1-62.

19. Sharma P, et al. Primary, adaptive, and acquired resistance to cancer immunotherapy. Cell. 2017;168(4):707-723.

20. Postow MA, et al. Immune-related adverse events associated with immune checkpoint blockade. N Engl J Med. 2018;378(2):158-168.

21. Snyder A, et al. Genetic basis for clinical response to CTLA-4 blockade in melanoma. $N$ Engl J Med. 2014;371(23):2189-2199.

22. Rizvi NA, et al. Cancer immunology. Mutational landscape determines sensitivity to PD-1 blockade in non-small cell lung cancer. Science. 2015;348(6230):124-128.

23. Goodman AM, et al. Tumor mutational burden as an independent predictor of response to immunotherapy in diverse cancers. Mol Cancer Ther. 2017;16(11):2598-2608.

24. Haanen J. Converting cold into hot tumors by combining immunotherapies. Cell. 2017;170(6):1055-1056.

25. Hellmann MD, et al. Tumor mutational burden and efficacy of nivolumab monotherapy and in combination with ipilimumab in small-cell lung cancer. Cancer Cell. 2018;33(5):853-861.

26. Arozarena I, Wellbrock C. Phenotype plasticity as enabler of melanoma progression and therapy resistance. Nat Rev Cancer. 2019;19(7):377-391.

27. Hoek KS, et al. In vivo switching of human melanoma cells between proliferative and invasive states. Cancer Res. 2008;68(3):650-656.

28. Landsberg J, et al. Melanomas resist T-cell therapy through inflammation-induced reversible dedifferentiation. Nature. 2012;490(7420):412-416.

29. Mehta A, et al. Immunotherapy resistance by inflammation-induced dedifferentiation. Cancer Discov. 2018;8(8):935-943.

30. Tsoi J, et al. Multi-stage differentiation defines melanoma subtypes with differential vulnerability to drug-induced iron-dependent oxidative stress. Cancer Cell. 2018;33(5):890-904.

31. Spranger $S$, et al. Melanoma-intrinsic $\beta$-catenin signalling prevents anti-tumour immunity.
Nature. 2015;523(7559):231-235.

32. Restivo G, et al. The low affinity neurotrophin receptor CD271 regulates phenotype switching in melanoma. Nat Commun. 2017;8(1):1988.

33. Redmer $\mathrm{T}$, et al. The nerve growth factor receptor CD271 is crucial to maintain tumorigenicity and stem-like properties of melanoma cells. PLoS One. 2014;9(5):e92596.

34. Riesenberg S, et al. MITF and c-Jun antagonism interconnects melanoma dedifferentiation with pro-inflammatory cytokine responsiveness and myeloid cell recruitment. Nat Commun. 2015;6:8755.

35. Zhu J, et al. Resistance to cancer immunotherapy mediated by apoptosis of tumor-infiltrating lymphocytes. Nat Commun. 2017;8(1):1404.

36. Dankort D, et al. Braf(V600E) cooperates with Pten loss to induce metastatic melanoma. Nat Genet. 2009;41(5):544-552.

37. Hooijkaas AI, et al. Targeting BRAFV600E in an inducible murine model of melanoma. Am J Pathol. 2012;181(3):785-794.

38. Levy C, et al. MITF: master regulator of melanocyte development and melanoma oncogene. Trends Mol Med. 2006;12(9):406-414.

39. Kawakami Y, et al. Cloning of the gene coding for a shared human melanoma antigen recognized by autologous T cells infiltrating into tumor. Proc Natl Acad Sci U S A .1994;91(9):3515-3519.

40. Coulie PG, et al. A new gene coding for a differentiation antigen recognized by autologous cytolytic T lymphocytes on HLA-A2 melanomas. JExp Med.1994;180(1):35-42.

41. Jenkins MH, et al. Multiple murine BRaf(V600E) melanoma cell lines with sensitivity to PLX4032. Pigment Cell Melanoma Res. 2014;27(3):495-501.

42. Damsky WE, et al. $\beta$-Catenin signaling controls metastasis in Braf-activated Pten-deficient melanomas. Cancer Cell. 2011;20(6):741-754.

43. Hoek KS, Goding CR. Cancer stem cells versus phenotype-switching in melanoma. Pigment Cell Melanoma Res. 2010;23(6):746-759.

44. Kemper K, et al. Phenotype switching: tumor cell plasticity as a resistance mechanism and target for therapy. Cancer Res. 2014;74(21):5937-5941.

45. Verfaillie A, et al. Decoding the regulatory landscape of melanoma reveals TEADS as regulators of the invasive cell state. Nat Commun. 2015;6:6683.

46. Hoek KS, et al. Metastatic potential of melanomas defined by specific gene expression pro- 
files with no BRAF signature. Pigment Cell Res. 2006;19(4):290-302.

47. Gandin V, et al. nanoCAGE reveals 5' UTR features that define specific modes of translation of functionally related MTOR-sensitive mRNAs. Genome Res. 2016;26(5):636-648.

48. Fallahi-Sichani M, et al. Adaptive resistance of melanoma cells to RAF inhibition via reversible induction of a slowly dividing de-differentiated state. Mol Syst Biol. 2017;13(1):905.

49. Folberg R, et al. Tumor cell plasticity in uveal melanoma: microenvironment directed dampening of the invasive and metastatic genotype and phenotype accompanies the generation of vasculogenic mimicry patterns. Am J Pathol. 2006;169(4):1376-1389.

50. Reinhardt J, et al. MAPK signaling and inflammation link melanoma phenotype switching to induction of $\mathrm{CD} 73$ during immunotherapy. Cancer Res. 2017;77(17):4697-4709.

51. Bronte $\mathrm{V}$, et al. Recommendations for myeloid-derived suppressor cell nomenclature and characterization standards. Nat Commun. 2016;7:12150.

52. Gabrilovich DI, Nagaraj S. Myeloid-derived suppressor cells as regulators of the immune system. Nat Rev Immunol. 2009;9(3):162-174.

53. Lechner MG, et al. Characterization of cytokine-induced myeloid-derived suppressor cells from normal human peripheral blood mononuclear cells. J Immunol. 2010;185(4):2273-2284.

54. Peng $\mathrm{W}$, et al. Loss of PTEN promotes resistance to T cell-mediated immunotherapy. Cancer Discov. 2016;6(2):202-216.

55. Aldinucci D, Colombatti A. The inflammatory chemokine CCL5 and cancer progression. Mediators Inflamm. 2014;2014:292376.

56. Ban Y, et al. Targeting autocrine CCL5-CCR5 axis reprograms immunosuppressive myeloid cells and reinvigorates antitumor immunity. Cancer Res. 2017;77(11):2857-2868.

57. Wang J, et al. UV-induced somatic mutations elicit a functional $\mathrm{T}$ cell response in the YUMMER1.7 mouse melanoma model. Pigment Cell Melanoma Res. 2017;30(4):428-435.

58. Ferrari de Andrade L, et al. Natural killer cells are essential for the ability of BRAF inhibitors to control BRAFV600E-mutant metastatic melanoma.
Cancer Res. 2014;74(24):7298-7308.

59. Xu Y, et al. Translation control of the immune checkpoint in cancer and its therapeutic targeting. Nat Med.2019;25(2):301-311.

60. Cerezo M, et al. Translational control of tumor immune escape via the eIF4F-STAT1-PD-L1 axis in melanoma. Nat Med. 2018;24(12):1877-1886.

61. Oh SA, et al. PD-L1 expression by dendritic cells is a key regulator of T-cell immunity in cancer. Nat Cancer. 2020;1(7):681-691.

62. Peng Q, et al. PD-L1 on dendritic cells attenuates $\mathrm{T}$ cell activation and regulates response to immune checkpoint blockade. Nat Commun. 2020;11(1):4835.

63. Lau J, et al. Tumour and host cell PD-L1 is required to mediate suppression of anti-tumour immunity in mice. Nat Commun. 2017;8:14572.

64. Weber R, et al. Myeloid-derived suppressor cells hinder the anti-cancer activity of immune checkpoint inhibitors. Front Immunol. 2018;9:1310.

65. Frederick DT, et al. BRAF inhibition is associated with enhanced melanoma antigen expression and a more favorable tumor microenvironment in patients with metastatic melanoma. Clin Cancer Res. 2013;19(5):1225-1231.

66. Hugo W, et al. Genomic and transcriptomic features of response to anti-PD-1 therapy in metastatic melanoma. Cell. 2016;165(1):35-44.

67. Cooper ZA, et al. Response to BRAF inhibition in melanoma is enhanced when combined with immune checkpoint blockade. Cancer Immunol Res. 2014;2(7):643-654.

68. Robichaud N, et al. Translational control in the tumor microenvironment promotes lung metastasis: Phosphorylation of eIF4E in neutrophils. Proc Natl Acad Sci U S A. 2018;115(10):E2202-E2209.

69. Guo Q, et al. MNK1/NODAL signaling promotes invasive progression of breast ductal carcinoma in situ. Cancer Res. 2019;79(7):1646-1657.

70. Kumar K, et al. Differential regulation of ZEB1 and EMT by MAPK-interacting protein kinases (MNK) and eIF4E in pancreatic cancer. Mol Cancer Res. 2016;14(2):216-227.

71. Bartish $\mathrm{M}$, et al. MNK2 governs the macrophage antiinflammatory phenotype. Proc Natl Acad Sci US A. 2020;117(44):27556-27565.

72. Salmon H, et al. Expansion and activation of CD103(+) dendritic cell progenitors at the tumor site enhances tumor responses to therapeutic PD-L1 and BRAF inhibition. Immunity. 2016;44(4):924-938.

73. Williford JM, et al. Recruitment of $\mathrm{CD}_{103^{+}}$dendritic cells via tumor-targeted chemokine delivery enhances efficacy of checkpoint inhibitor immunotherapy. Sci Adv. 2019;5(12):eaay1357.

74. Lim SY, et al. Circulating cytokines predict immune-related toxicity in melanoma patients receiving anti-PD-1-based immunotherapy. Clin Cancer Res. 2019;25(5):1557-1563.

75. Hsu J, et al. Contribution of NK cells to immunotherapy mediated by PD-1/PD-L1 blockade. J Clin Invest. 2018;128(10):4654-4668.

76. Siddiqui I, et al. Intratumoral Tcf1 ${ }^{+} \mathrm{PD}-1^{+} \mathrm{CD} 8^{+} \mathrm{T}$ cells with stem-like properties promote tumor control in response to vaccination and checkpoint blockade immunotherapy. Immunity. 2019;50(1):195-211.

77. Miller BC, et al. Subsets of exhausted CD8(+) T cells differentially mediate tumor control and respond to checkpoint blockade. Nat Immunol. 2019;20(3):326-336.

78. Lee LJ, et al. Cancer plasticity: the role of mRNA translation. Trends Cancer. 2021;7(2):134-145.

79. Terry S, et al. New insights into the role of EMT in tumor immune escape. Mol Oncol. 2017;11(7):824-846.

80. Simon S, et al. Emergence of high-avidity melan-A-specific clonotypes as a reflection of anti-PD-1 clinical efficacy. Cancer Res. 2017;77(24):7083-7093.

81. Boni A, et al. Selective BRAFV600E inhibition enhances T-cell recognition of melanoma without affecting lymphocyte function. Cancer Res. 2010;70(13):5213-5219.

82. Hu-Lieskovan S, et al. Improved antitumor activity of immunotherapy with BRAF and MEK inhibitors in BRAF(V600E) melanoma. Sci Transl Med.2015;7(279):279ra41.

83. Wilmott JS, et al. Selective BRAF inhibitors induce marked T-cell infiltration into human metastatic melanoma. Clin Cancer Res. 2012;18(5):1386-1394.

84. Ribas A, et al. Combined BRAF and MEK inhibition with PD-1 blockade immunotherapy in BRAF-mutant melanoma. Nat Med. 2019;25(6):936-940. 